Article

\title{
Synthesis and Antibacterial Activity of Amino Acid and Dipeptide Prodrugs of IMB-070593, a Fluoroquinolone Candidate
}

\author{
Tingting Zhang ${ }^{1,2, \dagger}$, Jinwei Wu ${ }^{2, \dagger}$, Shihong Chen ${ }^{2}$, Kaixiang Liu ${ }^{3}$, Yabin Lin ${ }^{4}$, Huiyuan Guo ${ }^{1}$ \\ and Mingliang Liu ${ }^{1, *}$
}

1 Institute of Medicinal Biotechnology, Chinese Academy of Medical Sciences and Peking Union Medical College, Beijing 100050, China

2 Zhejiang Starry Pharmaceutical Co. Ltd., Xianju 317300, China

3 China Resources Double-Crane Pharmaceutical Co. Ltd., Beijing 100102, China

4 Harbin Stomatological Hospital, Harbin 150010, China

$\dagger$ These authors contributed equally to this work.

* Author to whom correspondence should be addressed; E-Mail: lmllyx@126.com; Tel./Fax: +86-10-6303-6965.

Received: 14 April 2014; in revised form: 20 May 2014 / Accepted: 21 May 2014 /

Published: 23 May 2014

\begin{abstract}
A series of amino acid and dipeptide prodrugs of IMB-070593, a fluoroquinolone candidate discovered in our lab, were synthesized and evaluated for their water solubility and then antibacterial activity. Our results reveal that four amino acid prodrugs 4a,b,e,f and two dipeptide prodrugs $\mathbf{4 k}, \mathbf{l}$ have much greater solubility $(>85 \mathrm{mg} / \mathrm{mL})$ than IMB-070593 mesylate $(22.5 \mathrm{mg} / \mathrm{mL})$. Compounds $\mathbf{4 a}$ and $\mathbf{4 k}$ show good in vivo efficacy against MSSA $12-1$ (p.o./i.v., $5.32-7.68 \mathrm{mg} / \mathrm{kg}$ ) and $S$. pneumoniae $12-10$ (p.o., $18.39-23.13 \mathrm{mg} / \mathrm{kg}$ ) which is $1.19-1.50$ fold more active than the parent drug.
\end{abstract}

Keywords: IMB-070593; prodrugs; synthesis; water solubility; antibacterial activity

\section{Introduction}

Fluoroquinolone (FQ) antibacterial agents which target two type II bacterial topoisomerase enzymes, DNA gyrase and/or topoisomerase IV, are among the most attractive drugs in the antiinfective chemotherapy field [1]. 
The structure activity relationship (SAR) studies of FQs show that the basic substituent at the C-7 position, the most amenable site for chemical changes, greatly influences their antibacterial potency, spectrum and safety [2]. The presence of five- or six- membered nitrogen heterocycle including pyrrolidine, piperazine and piperidine at this position is a particularly favorable structural feature of important FQs on the market [3]. Moreover, methyloxime-functionalized pyrrolidines as novel C-7 substituents have also been proved to be of importance with respect to biological activity and led to the discovery of some new FQ agents, such as gemifloxacin, zabofloxacin and DW286 [4-6].

Recently, as a part of an ongoing program to optimize FQs against bacterial pathogens and mycobacterium tuberculosis (MTB), we have focused our attention on exploring the effect of introducing an oxime group into azetidine, pyrrolidine or piperidine side chains at the C-7 position of FQs, and some of them were found to have considerable biological activity [7-10]. For example, IMB070593 (Figure 1), a piperidinyl-based FQ candidate discovered in our lab and in late pre-clinical stage of development currently, possesses potent antibacterial and anti-MTB activity [9,11] as well as extremely low phototoxicity, hepatotoxicity and cardiac toxicity (unpublished data). However, IMB-070593 has low water solubility $(0.4 \mathrm{mg} / \mathrm{mL})$ and its mesylate salt still exhibits poor solubility $(22.5 \mathrm{mg} / \mathrm{mL})$. Since water solubility at physiological $\mathrm{pH}$ is important for preclinical testing, in vivo efficacy, and parenteral formulation [12], we decided to improve the solubility of IMB-070593 by employing a prodrug strategy.

Figure 1. Structure of IMB-070593.<smiles>CON=C1CCN(c2c(F)cc3c(=O)c(C(=O)O)cn(C4CC4)c3c2OC)CC1N</smiles>

One of the best strategies for increasing solubility of a drug containing a hydroxyl, carboxyl or an amino moiety is, in pharmaceutical field, to transform it into an amino acid prodrug, as exemplified by valaciclovir (a valine prodrug of acyclovir) [13]. We had previously reported that some of the amino acid prodrugs of tosufloxacin and moxifloxacin (MXFX) have better solubility and in vivo activity against clinically pathogens than the parent drugs [14,15].

Inspired by the above research results, it was decided to introduce common amino acids onto the nitrogen of the 3-amino-4-(methoxyimino)piperidine side chain of IMB-070593 by covalent binding. Thus, a series of amino acid and dipeptide prodrugs of IMB-070593 were designed, synthesized and evaluated for their water solubility and then antibacterial activity in this study. Our primary objective was to improve the solubility of IMB-070593, followed by optimizing its in vivo potency so as to finally develop the parenteral formulation. 


\section{Results and Discussion}

\subsection{Chemistry}

Synthetic pathways to the prodrugs $4 \mathbf{a}-\mathbf{l}$ of IMB-070593 are depicted in Scheme 1. According to published procedures [14,15], treatment of various amino acids $\mathbf{1 a}-\mathbf{j}$ with di-tert-butyl dicarbonate $\left(\mathrm{Boc}_{2} \mathrm{O}\right)$ in methanol gave $N$-Boc-protected acids $\mathbf{2 a}-\mathbf{j}$, which were coupled with IMB-070593 in the presence of dicyclohexylcarbodiimide (DCC) to yield the condensation products $\mathbf{3 a}-\mathbf{j}$. The $N$-Boc protecting group on $\mathbf{3 a}-\mathbf{j}$ was removed with trifluoroacetic acid (TFA) to provide the amino acid prodrugs $\mathbf{4 a - j}$. Similarly, Gly-Gly-amino prodrug $\mathbf{4 k}$ or L-Ala-L-Ala-amino prodrug $4 \mathbf{l}$ could be conveniently obtained from the corresponding mono-Gly-amino prodrug $\mathbf{4 a}$ and $N$-Boc-protected glycine $\mathbf{2 a}$ or mono-L-Ala-amino prodrug $\mathbf{4 b}$ and $N$-Boc-protected L-alanine $\mathbf{2 b}$ according to the same procedures used for preparation of $\mathbf{4 a}-\mathbf{j}$.

Scheme 1. Synthesis of the prodrugs $4 \mathbf{a}-\mathbf{l}$ of IMB-070593.
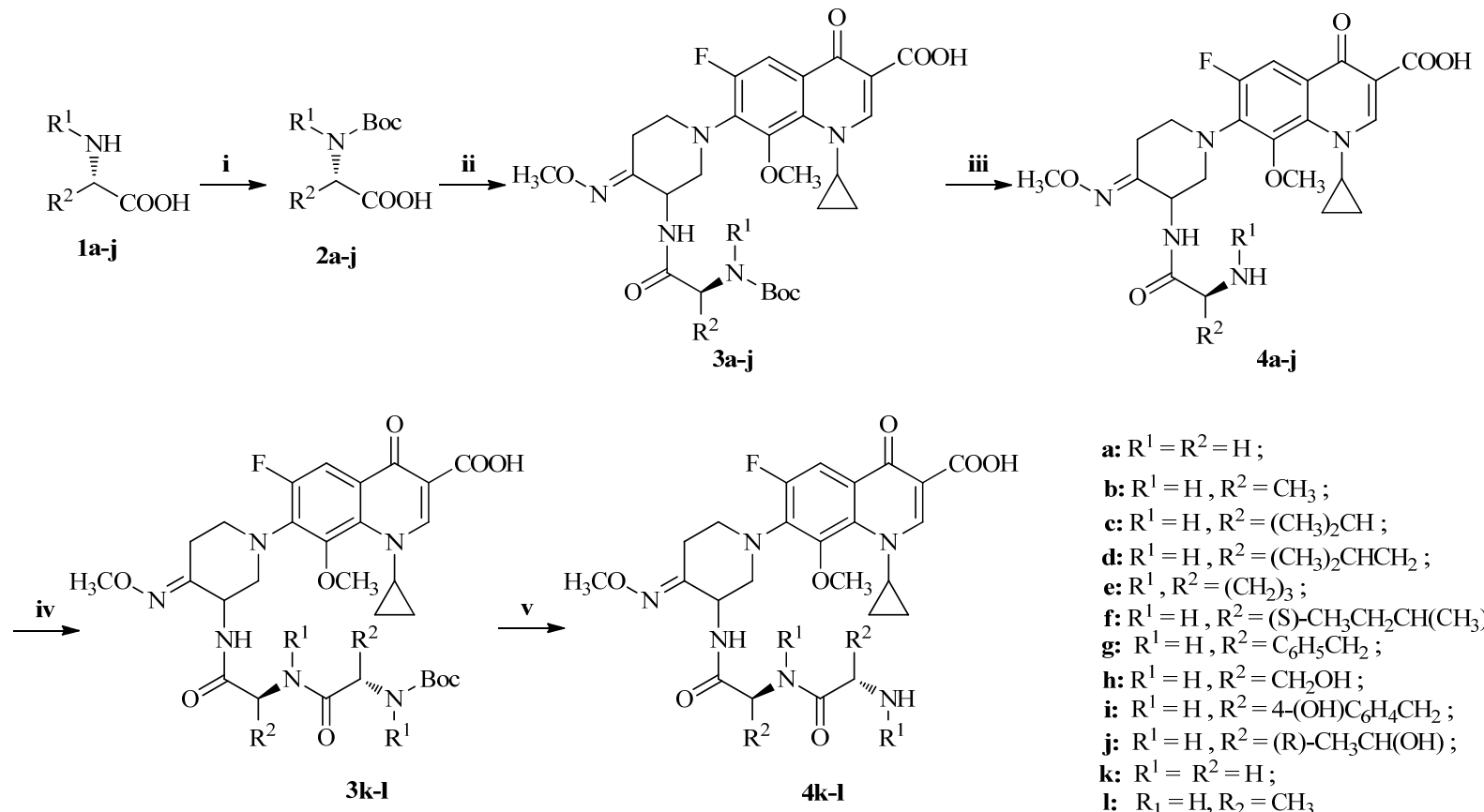

$$
\begin{aligned}
& \text { a: } \mathrm{R}^{1}=\mathrm{R}^{2}=\mathrm{H} ; \\
& \text { b: } \mathrm{R}^{1}=\mathrm{H}, \mathrm{R}^{2}=\mathrm{CH}_{3} ; \\
& \text { c: } \mathrm{R}^{1}=\mathrm{H}, \mathrm{R}^{2}=\left(\mathrm{CH}_{3}\right)_{2} \mathrm{CH} ; \\
& \text { d: } \mathrm{R}^{1}=\mathrm{H}, \mathrm{R}^{2}=\left(\mathrm{CH}_{3}\right)_{2} \mathrm{CHCH}_{2} ; \\
& \text { e: } \mathrm{R}^{1}, \mathrm{R}^{2}=\left(\mathrm{CH}_{2}\right)_{3} ; \\
& \text { f: } \mathrm{R}^{1}=\mathrm{H}, \mathrm{R}^{2}=\left(\mathrm{S}^{2}-\mathrm{CH}_{3} \mathrm{CH}_{2} \mathrm{CH}\left(\mathrm{CH}_{3}\right) ;\right. \\
& \text { g: } \mathrm{R}^{1}=\mathrm{H}, \mathrm{R}^{2}=\mathrm{C}_{6} \mathrm{H}_{5} \mathrm{CH}_{2} ; \\
& \text { h: } \mathrm{R}^{1}=\mathrm{H}, \mathrm{R}^{2}=\mathrm{CH}_{2} \mathrm{OH} ; \\
& \text { i: } \mathrm{R}^{1}=\mathrm{H}, \mathrm{R}^{2}=4-(\mathrm{OH}) \mathrm{C}_{6} \mathrm{H}_{4} \mathrm{CH}_{2} ; \\
& \text { j: } \mathrm{R}^{1}=\mathrm{H}, \mathrm{R}^{2}=(\mathrm{R})-\mathrm{CH}_{3} \mathrm{CH}(\mathrm{OH}) ; \\
& \text { k: } \mathrm{R}^{1}=\mathrm{R}^{2}=\mathrm{H} ; \\
& \text { l: } \mathrm{R}_{1}=\mathrm{H}, \mathrm{R}_{2}=\mathrm{CH}_{3}
\end{aligned}
$$

Reagents and Conditions: (i) (1) (Boc) $)_{2} \mathrm{O},\left(\mathrm{C}_{2} \mathrm{H}_{5}\right)_{3} \mathrm{~N}, \mathrm{CH}_{3} \mathrm{OH}$, (2) $2 \mathrm{~N} \mathrm{HCl}$; (ii) IMB-070593, DCC, $\mathrm{CH}_{2} \mathrm{Cl}_{2}$; (iii)/(v) (1) TFA, (2) $\mathrm{NH}_{4} \mathrm{OH}$; (iv) $2 \mathbf{a}$ or $\mathbf{2 b}, \mathrm{DCC}, \mathrm{CH}_{2} \mathrm{Cl}_{2}$.

Because the methyloxime group of all the prodrugs $4 \mathbf{a}-\mathbf{l}$ may be present in the $E$ - or $Z$ configuration, it was necessary to determine their geometries. Although we were unable to prepare X-ray quality single crystals of any methyloxime intermediate or product in this study, we had previously obtained the ingle crystals of 4-(methoxyimino)-3-methylaminopiperidine dihydrochloride, a $N$-methylated analogue of the side chain at C-7 position of IMB-070593, in which the piperidine ring adopts a chair conformation and the methyloxime geometry exists in an E-configuration [9]. Accordingly, we can speculate that the methyloxime group of the prodrugs in this study should have the same $E$-configuration. 


\subsection{Water Solubility}

The prodrugs $\mathbf{4 a}-\mathbf{l}$ were initially evaluated for their water solubility which was determined by HPLC measurement of the concentration of a micromembrane filtered saturated solution [16]. The solubility of 4a-l along with the parent IMB-070593 and IMB-070593 mesylate for comparison is presented in Table 1. The data reveal that all of $\mathbf{4 a}-\mathbf{l}$ have greater solubility $(0.6-795.1 \mathrm{mg} / \mathrm{mL})$ than that of IMB-070593 $(0.4 \mathrm{mg} / \mathrm{mL})$, and two dipeptide prodrugs $4 \mathbf{k}$ and $\mathbf{4 l}(235.0$ and $281.5 \mathrm{mg} / \mathrm{mL}$, respectively) are more soluble than the corresponding amino acid prodrugs $\mathbf{4 a}$ and $\mathbf{4 b}$ (97.0 and $112.0 \mathrm{mg} / \mathrm{mL}$, respectively). It is encouraging that prodrugs $\mathbf{4 a}, \mathbf{b}, \mathbf{e}, \mathbf{f}, \mathbf{k}, \mathbf{l}$ have also much greater solubility $(>85 \mathrm{mg} / \mathrm{mL})$ than the parent IMB-070593 mesylate $(22.5 \mathrm{mg} / \mathrm{mL})$ which suggest that the six prodrugs could display better in vivo efficacy than IMB-070593 mesylate.

Table 1. Structures, physical data and solubility of compounds $\mathbf{4 a - 1 .}$

\begin{tabular}{|c|c|c|c|c|c|}
\hline Compd. & $\mathbf{R}^{1}$ & $\mathbf{R}^{2}$ & $\begin{array}{c}\text { m.p. } \\
{\left[{ }^{\circ} \mathbf{C}\right]^{a}}\end{array}$ & {$[\boldsymbol{\alpha}]_{D}^{20}\left(\mathbf{c}, \mathbf{C H}_{3} \mathbf{O H}\right)$} & $\begin{array}{c}\text { Water } \\
\text { Solubility } \\
(\mathrm{mg} / \mathrm{mL})\end{array}$ \\
\hline $4 a$ & $\mathrm{H}$ & $\mathrm{H}$ & $136-137$ & $0^{\circ}(0.50)$ & 112.0 \\
\hline $4 b$ & $\mathrm{H}$ & $\mathrm{CH}_{3}$ & $133-134$ & $-2.80^{\circ}(0.50)$ & 97.0 \\
\hline $4 c$ & $\mathrm{H}$ & $\left(\mathrm{CH}_{3}\right)_{2} \mathrm{CH}$ & $154-156$ & $-24.30^{\circ}(0.50)$ & 15.6 \\
\hline 4d & $\mathrm{H}$ & $\left(\mathrm{CH}_{3}\right)_{2} \mathrm{CHCH}_{2}$ & $109-110$ & $-20.37^{\circ}(0.11)$ & 5.3 \\
\hline $4 e$ & & $\left(\mathrm{CH}_{2}\right)_{3}$ & $157-158$ & $-19.17^{\circ}(0.24)$ & 795.1 \\
\hline $4 f$ & $\mathrm{H}$ & (S) $-\mathrm{CH}_{3} \mathrm{CH}_{2} \mathrm{CH}\left(\mathrm{CH}_{3}\right)$ & $136-138$ & $-26.68^{\circ}(0.40)$ & 87.4 \\
\hline $4 g$ & $\mathrm{H}$ & $\mathrm{C}_{6} \mathrm{H}_{5} \mathrm{CH}_{2}$ & $123-125$ & $-13.32^{\circ}(0.77)$ & 1.0 \\
\hline $4 h$ & $\mathrm{H}$ & $\mathrm{CH}_{2} \mathrm{OH}$ & $114-116$ & $-9.01^{\circ}(0.44)$ & 50.2 \\
\hline $4 i$ & $\mathrm{H}$ & 4-(OH) $\mathrm{C}_{6} \mathrm{H}_{4} \mathrm{CH}_{2}$ & $138-140$ & $-14.14^{\circ}(0.50)$ & 0.6 \\
\hline $4 j$ & $\mathrm{H}$ & (R)- $\mathrm{CH}_{3} \mathrm{CH}(\mathrm{OH})$ & $111-113$ & $-15.25^{\circ}(0.12)$ & 72.3 \\
\hline $4 k$ & $\mathrm{H}$ & $\mathrm{H}$ & $121-123$ & $0^{\circ}(0.13)$ & 235.0 \\
\hline 41 & $\mathrm{H}$ & $\mathrm{CH}_{3}$ & $120-121$ & $-27.19^{\circ}(0.23)$ & 281.5 \\
\hline IMB & & & & & 0.4 \\
\hline IMB mesylate & & & & & 22.5 \\
\hline
\end{tabular}

${ }^{a}$ Melting points are uncorrected; IMB: IMB-070593.

\subsection{Antibacterial Activity}

\subsubsection{In Vitro Activity}

The prodrugs $4 \mathbf{a}-\mathbf{l}$ were evaluated for their in vitro antibacterial activity against representative bacterial strains using standard techniques [17]. Minimum inhibitory concentration (MIC) is defined as the concentration of the compound required to give complete inhibition of bacterial growth, and MIC values of 4a-l against Gram-positive and Gram-negative strains along with IMB-070593 mesylate, MXFX and levofloxacin (LVFX) for comparison, are listed in Table 2. 
Table 2. In vitro activity of compounds $\mathbf{4 a - 1}$ against Gram-positive and Gram-negative strains.

\begin{tabular}{|c|c|c|c|c|c|c|c|c|c|c|c|c|c|c|c|}
\hline \multirow[t]{2}{*}{ Compd. } & \multicolumn{15}{|c|}{ Strains MIC $(\mu \mathrm{g} / \mathrm{mL})$} \\
\hline & S.a. & MSSA & MRSA & MSSE & MRSE & S.p. & E.coli & E.co.1 & E.co.2 & E.co.3 & K.p.1 & K.p.2 & P.a.1 & P.a.2 & P.a.3 \\
\hline $4 a$ & 0.25 & 0.5 & 32 & 1 & 32 & 0.5 & 4 & 16 & 128 & $>128$ & 8 & 32 & 32 & 16 & 64 \\
\hline $4 b$ & 0.125 & 0.25 & 64 & 1 & 32 & 1 & 4 & 16 & 128 & $>128$ & 8 & 64 & 64 & 16 & 64 \\
\hline $4 c$ & 0.25 & 0.25 & 64 & 2 & 64 & 1 & 4 & 16 & $>128$ & $>128$ & 16 & 32 & 32 & 32 & 64 \\
\hline $4 d$ & 0.25 & 0.25 & 64 & 4 & 64 & 2 & 4 & 16 & $>128$ & $>128$ & 16 & 128 & 128 & 64 & 64 \\
\hline $4 e$ & 0.25 & 2 & 64 & 4 & 64 & 2 & 4 & 32 & $>128$ & $>128$ & 16 & 64 & 64 & 128 & 128 \\
\hline $4 f$ & 0.5 & 0.5 & 64 & 2 & 64 & 1 & 8 & 32 & $>128$ & $>128$ & 16 & 128 & 128 & 32 & 64 \\
\hline $4 \mathrm{~g}$ & 0.125 & 0.25 & 64 & 1 & 64 & 1 & 16 & 64 & $>128$ & $>128$ & 64 & $>128$ & 128 & 64 & 128 \\
\hline $4 h$ & 1 & 1 & 64 & 2 & 64 & 1 & 16 & 128 & $>128$ & $>128$ & 32 & $>128$ & 128 & 32 & 128 \\
\hline $4 i$ & 2 & 2 & $>128$ & 8 & 64 & 2 & 16 & 128 & $>128$ & $>128$ & 32 & $>128$ & 128 & 32 & 128 \\
\hline $4 j$ & 2 & 1 & 128 & 2 & 64 & 2 & 16 & 64 & $>128$ & $>128$ & 32 & 64 & 64 & 32 & 64 \\
\hline $4 k$ & 4 & 8 & $>128$ & 16 & 64 & 8 & 16 & 128 & $>128$ & $>128$ & 64 & $>128$ & $>128$ & $>128$ & 128 \\
\hline 41 & 4 & 4 & $>128$ & 32 & 64 & 8 & 32 & $>128$ & $>128$ & $>128$ & 64 & $>128$ & 128 & $>128$ & 128 \\
\hline IMB mesylate & $<0.008$ & $<0.008$ & 2 & 0.06 & 0.125 & 0.03 & 0.06 & 0.5 & 16 & 16 & 0.06 & 0.5 & 4 & 2 & 16 \\
\hline MXFX & $<0.008$ & $<0.008$ & 8 & 0.125 & 0.25 & 0.06 & $<0.008$ & 0.5 & 16 & 16 & 0.03 & 0.5 & 2 & 16 & 8 \\
\hline LVFX & 0.06 & 0.06 & 64 & 0.5 & 0.03 & 0.125 & $<0.008$ & 0.25 & 4 & 16 & 0.03 & 0.5 & 0.5 & 32 & 8 \\
\hline
\end{tabular}

S.a.: S. aureus ATCC 25923. MSSA: Methicillin-sensitive S. aureus 12-4. MRSA: Methicillin-resistant S. aureus 12-1. MSSE: Methicillin-sensitive S.epidermidis 12-3. MRSE: Methicillin-resistant S. epidermidis 12-1. S.p.: S. pneumoniae ATCC 49619. E.coli: E. coli ATCC 25922. E.co.1: E. coli 12-6. E.co.2: E. coli 12-11. E.co.3: Extended-spectrum $\beta$-lactamase-producing $\left(\mathrm{ESBL}^{+}\right)$E. coli 12-14. K.p.1: K. pneumoniae 12-4. K.p.2: ESBL K. pneumoniae 12-7. P.a.1: P. aeruginosa 12-12. P.a.2: P. aeruginosa 12-14. P.a.3: P. aeruginosa 12-20. IMB mesylate: IMB-070593 mesylate. MXFX: Moxifloxacin. LVFX: Levofloxacin. 
The prodrugs 4a-l show significantly less activity than the parent IMB-070593, MXFX and LVFX which is consistent with the biological character of common prodrugs. Moreover, $4 \mathbf{a}-\mathbf{I}$ are generally more active against Gram-positive strains except methicillin-resistant $S$. aureus (MRSA) and methicillin-resistant S. epidermidis (MRSE) than Gram-negative ones, and they have virtually no activity against all of the tested Gram-negative strains except for few exceptions.

\subsubsection{In Vivo Activity}

Mice protection tests were used to evaluate in vivo efficacy of the prodrugs $\mathbf{4 a}, \mathbf{b}, \mathbf{e}, \mathbf{f}, \mathbf{k}, \mathbf{l}$ with greater solubility than IMB-070593 mesylate, given the fact that there is no obvious difference among of $\mathbf{4 a - 1}$ with regard to their in vitro activity. The efficacy of them was initially tested against two clinical isolate strains (methicillin-sensitive $S$. aureus / MSSA 12-1, E. Coli 12-1), and then 4a of the amino acid prodrugs and $\mathbf{4 k}$ of the dipeptide ones with better efficacy were chosen for further evaluation their protective effects in vivo (p.o.) against other four clinical isolates and that (i.v.) against the above two strains, IMB-070593 mesylate was used as the control drug (Table 3).

The results illustrate that all of the six prodrugs have better oral activity against MSSA 12-1 (6.44$9.39 \mathrm{mg} / \mathrm{kg})$ than IMB-070593 mesylate $(9.40 \mathrm{mg} / \mathrm{kg})$, but weaker against E. coli $12-1$. Furthermore, 4a and 4k show stronger efficacy than the parent drug against $S$. pneumonia 12-10 (p.o., 18.39 and $23.13 \mathrm{mg} / \mathrm{kg}$, respectively) and MSSA 12-1 (i.v., 5.71 and $5.32 \mathrm{mg} / \mathrm{kg}$, respectively), but weaker oral activity against MRSE 12-1, MRSA $12-5$ and $K$. pneumonia $12-1$. In a word, the prodrugs seem to be more active against drug-sensitive Gram-positive strains (p.o. or i.v., such as MSSA12-1 and $S$. pneumonia 12-10) than IMB-070593 mesylate. Conversely, they are much weaker against drugresistant Gram-positive strains (p.o., such as MRSE 12-1and MRSA 12-5) and Gram-negative strains (p.o., such as E. Coli 12-1 and K. pneumonia 12-1), compared with the parent drug.

Table 3. In vivo efficacy of selected compounds against systemic infections in mice.

\begin{tabular}{|c|c|c|c|c|}
\hline \multirow{3}{*}{$\begin{array}{c}\text { Infected strain } \\
(\mathbf{c f u} / \mathbf{m L}) \\
\text { MSSA } 12-1 \\
\left(3.0 \times 10^{4}\right)\end{array}$} & \multirow{2}{*}{$\begin{array}{c}\text { Compd } \\
4 a\end{array}$} & \multirow{2}{*}{$\begin{array}{c}\text { MIC } \\
(\mu \mathrm{g} / \mathbf{m L})\end{array}$} & \multicolumn{2}{|c|}{$\begin{array}{c}\mathrm{ED}_{50}(\mathrm{mg} / \mathrm{kg})^{\mathrm{a}} \\
{[95 \% \text { confidence limit }(\mathrm{mg} / \mathrm{kg})]}\end{array}$} \\
\hline & & & $7.16(10.13-4.97)^{b}$ & $5.71(7.97-4.11)^{\mathrm{c}}$ \\
\hline & $4 b$ & 0.5 & $9.30(13.43-6.41)$ & $\mathrm{NT}^{\mathrm{d}}$ \\
\hline \multirow{12}{*}{$\begin{array}{l}\text { E. coli } 12-1 \\
\left(6.0 \times 10^{5}\right)\end{array}$} & $4 e$ & 2 & $8.74(12.17-6.27)$ & NT \\
\hline & $4 f$ & 1 & $6.44(9.75-3.83)$ & NT \\
\hline & $4 k$ & 8 & $7.68(10.76-5.42)$ & $5.32(7.58-3.74)$ \\
\hline & 41 & 8 & $9.39(13.85-6.31)$ & NT \\
\hline & IMB mesylate & $<0.008$ & $9.40(13.36-6.60)$ & $7.15(10.73-4.95)$ \\
\hline & $4 \mathbf{a}$ & 16 & $17.53(25.25-12.04)$ & $12.51(19.41-8.37)$ \\
\hline & $4 b$ & 16 & $25.17(37.89-17.12)$ & NT \\
\hline & $4 e$ & 32 & $21.44(31.18-14.83)$ & NT \\
\hline & $4 f$ & 32 & $17.53(25.25-12.04)$ & NT \\
\hline & $4 k$ & 128 & $18.80(26.72-13.20)$ & $14.30(21.46-9.91)$ \\
\hline & 41 & $>128$ & $23.43(36.23-15.57)$ & NT \\
\hline & IMB mesylate & 0.5 & $8.76(12.63-6.02)$ & $7.67(11.83-5.27)$ \\
\hline
\end{tabular}


Table 3. Cont.

\begin{tabular}{|c|c|c|c|c|}
\hline \multirow{3}{*}{$\begin{array}{c}\begin{array}{c}\text { Infected strain } \\
(\mathbf{c f u} / \mathbf{m L} \mathbf{)}\end{array} \\
\text { MRSE } 12-1 \\
\left(4.5 \times 10^{6}\right)\end{array}$} & \multirow{2}{*}{$\begin{array}{c}\text { Compd } \\
4 a\end{array}$} & \multirow{2}{*}{$\begin{array}{c}\text { MIC } \\
(\boldsymbol{\mu g} / \mathbf{m L})\end{array}$} & \multicolumn{2}{|c|}{$\begin{array}{c}\mathrm{ED}_{50}(\mathrm{mg} / \mathrm{kg})^{\mathrm{a}} \\
{[95 \% \text { confidence limit }(\mathrm{mg} / \mathrm{kg})]}\end{array}$} \\
\hline & & & $50.55(39.57-66.50)$ & NT \\
\hline & $4 k$ & 64 & $54.03(40.89-77.35)$ & NT \\
\hline & IMB mesylate & 2 & $15.33(11.47-20.06)$ & NT \\
\hline & $4 a$ & 32 & $72.80(55.03-123.15)$ & NT \\
\hline \multirow[t]{2}{*}{$\left(4.5 \times 10^{6}\right)$} & $4 k$ & $>128$ & $86.06(67.10-152.78)$ & NT \\
\hline & IMB mesylate & 2 & $25.36(20.10-32.68)$ & NT \\
\hline S.pneumonia12-10 & $4 a$ & 1 & $18.39(14.00-23.60)$ & NT \\
\hline \multirow[t]{2}{*}{$\left(5.2 \times 10^{8}\right)$} & $4 \mathbf{k}$ & 2 & $23.13(17.30-31.81)$ & NT \\
\hline & IMB mesylate & 0.06 & $27.57(21.85-36.07)$ & NT \\
\hline K.pneumoniae 12-1 & $4 a$ & 4 & $33.91(26.82-44.56)$ & NT \\
\hline \multirow[t]{2}{*}{$\left(4.5 \times 10^{6}\right)$} & $4 k$ & 16 & $33.30(25.16-47.78)$ & NT \\
\hline & IMB mesylate & 0.06 & $18.56(14.55-23.97)$ & NT \\
\hline
\end{tabular}

\section{Experimental}

\subsection{General Information}

Melting points were determined in open capillaries and are uncorrected. ${ }^{1} \mathrm{H}-\mathrm{NMR}$ spectra were determined on a Varian Mercury-400/500/600 spectrometer in DMSO- $d_{6}$ or $\mathrm{CDCl}_{3}$ using tetramethylsilane (TMS) as an internal standard. Electrospray ionization (ESI) mass spectra and high resolution mass spectra (HRMS) were obtained on a MDSSCIEX Q-Tap mass spectrometer and AccuTOF CS JMS-T100CS (JEOL) mass spectrometer. Unless otherwise noted, the reagents were obtained from commercial supplier and used without further purification. TLC was performed on silica gel plates (Merck, ART5554 60F254).

\subsection{Synthesis}

\subsubsection{General Procedure for the Synthesis of $\mathbf{3 a}-\mathbf{j}$}

To a solution of amino acids $1 \mathbf{a}-\mathbf{j}(20 \mathrm{mmol})$ in methanol $(90 \mathrm{~mL})$ was added di-tert-butyl carbonate $(8.73 \mathrm{~g}, 40 \mathrm{mmol})$ and triethylamine $(11.1 \mathrm{~mL}, 80 \mathrm{mmol})$. The reaction mixture was heated to refluxing and stirred for $3 \mathrm{~h}$ at the same temperature, and concentrated under reduced pressure. The residue was diluted with water $(40 \mathrm{~mL})$, adjusted to $\mathrm{pH} 2.0-3.0$ with $2 \mathrm{~N} \mathrm{HCl}$ at $0-5{ }^{\circ} \mathrm{C}$, and then extracted with ethyl acetate $(50 \mathrm{~mL} \times 3)$. The combined extracts were washed with saturated brine $(30 \mathrm{~mL})$, dried over anhydrous $\mathrm{Na}_{2} \mathrm{SO}_{4}$, and then concentrated under reduced pressure to provide $\mathbf{2 a}-\mathbf{j}$ as white solids [18-23] (64.8\%-91.4\%). A mixture of IMB-070593 (2.08 g, $4.97 \mathrm{mmol}), \mathbf{2 a}-\mathbf{j}$ (5.71 mmol), dicyclohexylcarbodiimide $(1.18 \mathrm{~g}, 5.71 \mathrm{mmol})$ and dry dichloromethane $(42 \mathrm{~mL})$ was stirred at room temperature for $1 \mathrm{~h}$ and filtered. The filtrate was concentrated under reduced pressure, and the residue was treated with diethyl ether $(20 \mathrm{~mL})$, and then filtered. The solid was purified by column 
chromatography (silica gel) eluted with dichloromethane and methanol (v:v $=55: 1)$ to afford the title compounds $\mathbf{3 a}-\mathbf{j}(36 \%-79 \%$, from $\mathbf{1 a}-\mathbf{j})$ as white or yellow solids.

7-(3-(2-(tert-Butoxycarbonylamino)acetamido)-4-(methoxyimino)piperidin-1-yl)-1-cyclopropyl-6fluoro-8-methoxy-4-oxo-1,4-dihydroquinoline-3-carboxylic acid (3a). Obtained from 2a and IMB070593 as a white solid (86.1\%), m.p.: $139-140{ }^{\circ} \mathrm{C} .{ }^{1} \mathrm{H}-\mathrm{NMR}\left(400 \mathrm{MHz}, \mathrm{CDCl}_{3}\right) \delta(\mathrm{ppm}) 14.68(\mathrm{~s}, 1 \mathrm{H}$, $\mathrm{COOH}), 8.83\left(\mathrm{~s}, 1 \mathrm{H}, \mathrm{C}_{2}-\mathrm{H}\right), 7.90\left(\mathrm{~d}, J=11.7 \mathrm{~Hz}, 1 \mathrm{H}, \mathrm{C}_{5}-\mathrm{H}\right), 7.12(\mathrm{~d}, J=5.4 \mathrm{~Hz}, 1 \mathrm{H}), 5.09$ (s, 1H), $4.70-4.59(\mathrm{~m}, 1 \mathrm{H}), 4.18-4.14(\mathrm{~m}, 1 \mathrm{H}), 4.04-4.01(\mathrm{~m}, 1 \mathrm{H}), 3.92\left(\mathrm{~s}, 3 \mathrm{H}, \mathrm{CH}_{3} \mathrm{O}-\mathrm{N}\right), 3.85(\mathrm{~d}, J=5.4 \mathrm{~Hz}$, $1 \mathrm{H}), 3.79\left(\mathrm{~s}, 3 \mathrm{H}, \mathrm{CH}_{3} \mathrm{O}-\mathrm{C}\right), 3.72(\mathrm{q}, J=6.9 \mathrm{~Hz}, 1 \mathrm{H}), 3.62(\mathrm{~d}, J=9.0 \mathrm{~Hz}, 1 \mathrm{H}), 3.34(\mathrm{~d}, J=14.2 \mathrm{~Hz}$, 1H), 3.26 (t, $J=11.9 \mathrm{~Hz}, 1 \mathrm{H}), 3.04$ (t, $J=10.3 \mathrm{~Hz}, 1 \mathrm{H}), 2.34-2.32(\mathrm{~m}, 1 \mathrm{H}), 1.47$ (s, 9H, Boc), 1.30 1.19 (m, 2H, cyclopropyl $\left.\mathrm{CH}_{2}\right), 1.12-0.91\left(\mathrm{~m}, 2 \mathrm{H}\right.$, cyclopropyl $\left.\mathrm{CH}_{2}\right)$. MS-ESI $(\mathrm{m} / \mathrm{z}): 576.31(\mathrm{M}+\mathrm{H})^{+}$.

7-(3-((S)-2-(tert-Butoxycarbonylamino)propanamido)-4-(methoxyimino)piperidin-1-yl)-1-cyclopropyl6-fluoro-8-methoxy-4-oxo-1,4-dihydroquinoline-3-carboxylic acid (3b). Obtained from $\mathbf{2 b}$ as a offwhite solid (84.6\%), m.p.: $150-152{ }^{\circ} \mathrm{C} .{ }^{1} \mathrm{H}-\mathrm{NMR}\left(400 \mathrm{MHz}, \mathrm{CDCl}_{3}\right) \delta(\mathrm{ppm}) 14.68(\mathrm{~s}, 1 \mathrm{H}, \mathrm{COOH})$, $8.83\left(\mathrm{~s}, 1 \mathrm{H}, \mathrm{C}_{2}-\mathrm{H}\right), 7.91\left(\mathrm{~d}, J=11.6 \mathrm{~Hz}, 1 \mathrm{H}, \mathrm{C}_{5}-\mathrm{H}\right), 7.27-7.07(\mathrm{~m}, 1 \mathrm{H}), 4.98(\mathrm{~s}, 1 \mathrm{H}), 4.63(\mathrm{~s}, 1 \mathrm{H}), 4.21$ $(\mathrm{s}, 1 \mathrm{H}), 4.22-4.10(\mathrm{~m}, 1 \mathrm{H}), 4.03(\mathrm{~s}, 1 \mathrm{H}), 3.92\left(\mathrm{~d}, J=2.6 \mathrm{~Hz}, 3 \mathrm{H}, \mathrm{CH}_{3} \mathrm{O}-\mathrm{N}\right), 3.81\left(\mathrm{~s}, 3 \mathrm{H}, \mathrm{CH}_{3} \mathrm{O}-\mathrm{C}\right)$, $3.61(\mathrm{~d}, J=7.7 \mathrm{~Hz}, 1 \mathrm{H}), 3.36-3.24(\mathrm{~m}, 2 \mathrm{H}), 3.03(\mathrm{t}, J=10.8 \mathrm{~Hz}, 1 \mathrm{H}), 2.32(\mathrm{~s}, 1 \mathrm{H}), 1.46(\mathrm{~d}, J=6.8 \mathrm{~Hz}$, 9H, Boc), 1.38-1.26 (m, 3H, $\left.\mathrm{CH}_{3}\right), 1.25-1.13$ (m, 2H, cyclopropyl $\left.\mathrm{CH}_{2}\right), 1.01$ (s, 2H, cyclopropyl $\left.\mathrm{CH}_{2}\right)$. MS-ESI $(\mathrm{m} / \mathrm{z}): 590.32(\mathrm{M}+\mathrm{H})^{+}$.

7-(3-((S)-2-(tert-Butoxycarbonylamino)-3-methylbutanamido)-4-(methoxyimino)piperidin-1-yl)-1cyclopropyl-6-fluoro-8-methoxy-4-oxo-1,4-dihydroquinoline-3-carboxylic acid (3c). Obtained from 2c as a white solid (78.6\%), m.p.: $130-131{ }^{\circ} \mathrm{C} .{ }^{1} \mathrm{H}-\mathrm{NMR}\left(400 \mathrm{MHz}, \mathrm{CDCl}_{3}\right) \delta(\mathrm{ppm}) 14.69(\mathrm{~s}, 1 \mathrm{H}$, $\mathrm{COOH}), 8.83\left(\mathrm{~s}, 1 \mathrm{H}, \mathrm{C}_{2}-\mathrm{H}\right), 7.91\left(\mathrm{~d}, J=11.6 \mathrm{~Hz}, 1 \mathrm{H}, \mathrm{C}_{5}-\mathrm{H}\right), 7.01-6.89(\mathrm{~m}, 1 \mathrm{H}), 5.06(\mathrm{~s}, 1 \mathrm{H})$, 4.73-4.58 (m, 1H), 4.19-4.08 (m, 1H), 4.07-4.01 (m, 1H), $3.92\left(\mathrm{~d}, J=8.3 \mathrm{~Hz}, 3 \mathrm{H}, \mathrm{CH}_{3} \mathrm{O}-\mathrm{N}\right), 3.80$ (s, $\left.3 \mathrm{H}, \mathrm{CH}_{3} \mathrm{O}-\mathrm{C}\right), 3.61(\mathrm{~s}, 1 \mathrm{H}), 3.48(\mathrm{q}, J=7.0 \mathrm{~Hz}, 1 \mathrm{H}), 3.39-3.20(\mathrm{~m}, 2 \mathrm{H}), 3.06-2.99(\mathrm{~m}, 1 \mathrm{H}), 2.31$ (s, $1 \mathrm{H}), 2.21-2.08\left(\mathrm{~m}, 1 \mathrm{H}, \mathrm{C} \underline{\mathrm{H}}\left(\mathrm{CH}_{3}\right)_{2}\right), 1.45(\mathrm{~d}, J=2.3 \mathrm{~Hz}, 9 \mathrm{H}, \mathrm{Boc}), 1.23-1.21\left(\mathrm{~m}, 2 \mathrm{H}\right.$, cyclopropyl $\left.\mathrm{CH}_{2}\right)$, 1.09-0.88 (m, 8H, cyclopropyl $\left.\mathrm{CH}_{2}, \mathrm{CH}\left(\mathrm{C}_{3}\right)_{2}\right)$. MS-ESI $(\mathrm{m} / \mathrm{z}): 618.65(\mathrm{M}+\mathrm{H})^{+}$.

7-(3-((S)-2-(tert-Butoxycarbonylamino)-4-methylpentanamido)-4-(methoxyimino)piperidin-1-yl)-1cyclopropyl-6-fluoro-8-methoxy-4-oxo-1,4-dihydroquinoline-3-carboxylic acid (3d). Obtained from 2d as a yellow solid (58.2\%), m.p.: $142-143{ }^{\circ} \mathrm{C} .{ }^{1} \mathrm{H}-\mathrm{NMR}\left(500 \mathrm{MHz}, \mathrm{CDCl}_{3}\right) \delta$ (ppm) $14.67(\mathrm{~s}, 1 \mathrm{H}$, $\mathrm{COOH}), 8.83\left(\mathrm{~s}, 1 \mathrm{H}, \mathrm{C}_{2}-\mathrm{H}\right), 7.91\left(\mathrm{~d}, J=11.6 \mathrm{~Hz}, 1 \mathrm{H}, \mathrm{C}_{5}-\mathrm{H}\right), 7.26-7.02(\mathrm{~m}, 1 \mathrm{H}), 4.88(\mathrm{~d}, J=19.8 \mathrm{~Hz}$, $1 \mathrm{H}), 4.64(\mathrm{~s}, 1 \mathrm{H}), 4.14(\mathrm{~d}, J=17.1 \mathrm{~Hz}, 1 \mathrm{H}), 4.06-4.00(\mathrm{~m}, 1 \mathrm{H}), 3.92\left(\mathrm{~d}, J=9.4 \mathrm{~Hz}, 3 \mathrm{H}, \mathrm{CH}_{3} \mathrm{O}-\mathrm{N}\right)$, $3.80\left(\mathrm{~d}, J=2.6 \mathrm{~Hz}, 3 \mathrm{H}, \mathrm{CH}_{3} \mathrm{O}-\mathrm{C}\right), 3.61(\mathrm{~s}, 1 \mathrm{H}), 3.46(\mathrm{~d}, J=10.3 \mathrm{~Hz}, 1 \mathrm{H}), 3.39-3.21(\mathrm{~m}, 2 \mathrm{H}), 3.03$ (t, $J=10.5 \mathrm{~Hz}, 1 \mathrm{H}), 2.38-2.24(\mathrm{~m}, 1 \mathrm{H}), 1.94(\mathrm{~d}, J=9.8 \mathrm{~Hz}, 1 \mathrm{H}), 1.70(\mathrm{~s}, 2 \mathrm{H}), 1.45(\mathrm{~d}, J=6.4 \mathrm{~Hz}, 9 \mathrm{H}$, Boc), 1.38-1.30 (m, 1H), $1.27(\mathrm{~d}, J=10.0 \mathrm{~Hz}, 1 \mathrm{H}), 1.21-1.13\left(\mathrm{~m}, 2 \mathrm{H}\right.$, cyclopropyl $\left.\mathrm{CH}_{2}\right), 1.04-1.00$ $(\mathrm{m}, 1 \mathrm{H}), 0.98-0.95(\mathrm{~m}, 5 \mathrm{H})$. MS-ESI $(\mathrm{m} / \mathrm{z}): 632.63(\mathrm{M}+\mathrm{H})^{+}$.

7-(3-((S)-1-(tert-Butoxycarbonyl)pyrrolidine-2-carboxamido)-4-(methoxyimino)piperidin-1-yl)-1cyclopropyl-6-fluoro-8-methoxy-4-oxo-1,4-dihydroquinoline-3-carboxylic acid (3e). The title compound 3e was obtained from $2 \mathbf{e}$ as a off-white solid (83.6\%), m.p.: $186-187{ }^{\circ} \mathrm{C} .{ }^{1} \mathrm{H}-\mathrm{NMR}(400 \mathrm{MHz}$, 
$\left.\mathrm{CDCl}_{3}\right) \delta(\mathrm{ppm}) 14.67(\mathrm{~s}, 1 \mathrm{H}, \mathrm{COOH}), 8.83\left(\mathrm{~s}, 1 \mathrm{H}, \mathrm{C}_{2}-\mathrm{H}\right), 7.91\left(\mathrm{~d}, J=11.5 \mathrm{~Hz}, 1 \mathrm{H}, \mathrm{C}_{5}-\mathrm{H}\right), 7.00(\mathrm{~s}, 1 \mathrm{H})$, $4.63(\mathrm{~s}, 1 \mathrm{H}), 4.22(\mathrm{~s}, 1 \mathrm{H}), 4.10(\mathrm{~d}, J=15.4 \mathrm{~Hz}, 1 \mathrm{H}), 4.03(\mathrm{~d}, J=3.9 \mathrm{~Hz}, 1 \mathrm{H}), 3.91(\mathrm{~d}, J=2.6 \mathrm{~Hz}, 3 \mathrm{H}$, $\left.\mathrm{CH}_{3} \mathrm{O}-\mathrm{N}\right), 3.81$ (d, $\left.J=4.8 \mathrm{~Hz}, 3 \mathrm{H}, \mathrm{CH}_{3} \mathrm{O}-\mathrm{C}\right), 3.70-3.17(\mathrm{~m}, 6 \mathrm{H}), 3.03(\mathrm{~s}, 1 \mathrm{H}), 2.47-2.10(\mathrm{~m}, 2 \mathrm{H})$, 2.00-1.84 (m, 2H), $1.46(\mathrm{~d}, J=10.9 \mathrm{~Hz}, 9 \mathrm{H}, \mathrm{Boc}), 1.29-1.20\left(\mathrm{~m}, 2 \mathrm{H}\right.$, cyclopropyl $\left.\mathrm{CH}_{2}\right), 1.00(\mathrm{~d}$, $J=4.4 \mathrm{~Hz}, 2 \mathrm{H}$, cyclopropyl $\left.\mathrm{CH}_{2}\right)$. MS-ESI $(\mathrm{m} / \mathrm{z}): 616.67(\mathrm{M}+\mathrm{H})^{+}$.

7-(3-((2S,3S)-2-(tert-Butoxycarbonylamino)-3-methylpentanamido)-4-(methoxyimino)piperidin-1-yl)1-cyclopropyl-6-fluoro-8-methoxy-4-oxo-1,4-dihydroquinoline-3-carboxylic acid (3f). Obtained from 2f as a white solid (96.5\%), m.p. : $126-127{ }^{\circ} \mathrm{C} .{ }^{1} \mathrm{H}-\mathrm{NMR}\left(400 \mathrm{MHz}, \mathrm{CDCl}_{3}\right) \delta(\mathrm{ppm}) 14.68(\mathrm{~s}, 1 \mathrm{H}$, $\mathrm{COOH}), 8.83\left(\mathrm{~s}, 1 \mathrm{H}, \mathrm{C}_{2}-\mathrm{H}\right), 7.91\left(\mathrm{~d}, J=11.6 \mathrm{~Hz}, 1 \mathrm{H}, \mathrm{C}_{5}-\mathrm{H}\right), 6.99(\mathrm{~d}, J=5.9 \mathrm{~Hz}, 1 \mathrm{H}), 5.08(\mathrm{~s}, 1 \mathrm{H})$, $4.67(\mathrm{~d}, J=4.1 \mathrm{~Hz}, 1 \mathrm{H}), 4.21-3.98(\mathrm{~m}, 3 \mathrm{H}), 3.92\left(\mathrm{~d}, J=8.5 \mathrm{~Hz}, 3 \mathrm{H}, \mathrm{CH}_{3} \mathrm{O}-\mathrm{N}\right), 3.80\left(\mathrm{~s}, 3 \mathrm{H}, \mathrm{CH}_{3} \mathrm{O}-\mathrm{C}\right)$, $3.61(\mathrm{~s}, 1 \mathrm{H}), 3.35$ (d, $J=14.1 \mathrm{~Hz}, 1 \mathrm{H}), 3.26(\mathrm{~s}, 1 \mathrm{H}), 3.03(\mathrm{~d}, J=9.6 \mathrm{~Hz}, 1 \mathrm{H}), 2.30(\mathrm{~s}, 1 \mathrm{H}), 1.90-1.82$ (m, 2H), 1.46 (s, 9H, Boc), 1.29-1.16 (m, 3H), 1.01 (s, 2H), 0.95-0.91 (m, 6H). MS-ESI (m/z): 632.35 $(\mathrm{M}+\mathrm{H})^{+}$.

7-(3-((S)-2-(tert-Butoxycarbonylamino)-3-phenylpropanamido)-4-(methoxyimino)piperidin-1-yl)-1cyclopropyl-6-fluoro-8-methoxy-4-oxo-1,4-dihydroquinoline-3-carboxylic acid (3g). Obtained from $\mathbf{2 g}$ as a white solid (79.1\%), m.p.: $149-150{ }^{\circ} \mathrm{C} .{ }^{1} \mathrm{H}-\mathrm{NMR}\left(400 \mathrm{MHz}, \mathrm{CDCl}_{3}\right) \delta(\mathrm{ppm}) 14.68(\mathrm{~s}, 1 \mathrm{H}$, $\mathrm{COOH}), 8.84\left(\mathrm{~s}, 1 \mathrm{H}, \mathrm{C}_{2}-\mathrm{H}\right), 7.92\left(\mathrm{~d}, J=11.6 \mathrm{~Hz}, 1 \mathrm{H}, \mathrm{C}_{5}-\mathrm{H}\right), 7.40-7.22(\mathrm{~m}, 5 \mathrm{H}, \mathrm{ph}-\mathrm{H}), 6.90-6.77$ (m, $1 \mathrm{H}), 5.07-5.01(\mathrm{~m}, 1 \mathrm{H}), 4.56(\mathrm{~s}, 1 \mathrm{H}), 4.40(\mathrm{~s}, 1 \mathrm{H}), 4.16-3.97(\mathrm{~m}, 2 \mathrm{H}), 3.85(\mathrm{~d}, J=10.0 \mathrm{~Hz}, 3 \mathrm{H}$, $\left.\mathrm{CH}_{3} \mathrm{O}-\mathrm{N}\right), 3.79\left(\mathrm{~s}, 3 \mathrm{H}, \mathrm{CH}_{3} \mathrm{O}-\mathrm{C}\right), 3.56(\mathrm{~s}, 1 \mathrm{H}), 3.31-2.75(\mathrm{~m}, 5 \mathrm{H}), 2.28(\mathrm{t}, J=12.4 \mathrm{~Hz}, 1 \mathrm{H}), 1.43$ (s, 9H, Boc), 1.28-1.20 (m, 2H, cyclopropyl $\left.\mathrm{CH}_{2}\right), 1.03\left(\mathrm{~d}, J=11.3 \mathrm{~Hz}, 2 \mathrm{H}\right.$, cyclopropyl $\left.\mathrm{CH}_{2}\right)$. MS-ESI $(\mathrm{m} / \mathrm{z}): 666.31(\mathrm{M}+\mathrm{H})^{+}$.

7-(3-((S)-2-(tert-Butoxycarbonylamino)-3-hydroxypropanamido)-4-(methoxyimino)piperidin-1-yl)-1cyclopropyl-6-fluoro-8-methoxy-4-oxo-1,4-dihydroquinoline-3-carboxylic acid (3h). Obtained from $\mathbf{2 h}$ as a off-white solid (83.3\%), m.p. : $133-135{ }^{\circ} \mathrm{C} .{ }^{1} \mathrm{H}-\mathrm{NMR}\left(400 \mathrm{MHz}, \mathrm{CDCl}_{3}\right) \delta(\mathrm{ppm}) 14.64(\mathrm{~s}, 1 \mathrm{H}$, $\mathrm{COOH}), 8.83\left(\mathrm{~s}, 1 \mathrm{H}, \mathrm{C}_{2}-\mathrm{H}\right), 7.92\left(\mathrm{~d}, J=11.6 \mathrm{~Hz}, 1 \mathrm{H}, \mathrm{C}_{5}-\mathrm{H}\right), 7.15(\mathrm{~s}, 1 \mathrm{H}), 5.48(\mathrm{~s}, 1 \mathrm{H}), 4.84-4.61(\mathrm{~m}$, $1 \mathrm{H}), 4.23(\mathrm{~s}, 1 \mathrm{H}), 4.17-3.97(\mathrm{~m}, 3 \mathrm{H}), 3.93\left(\mathrm{~d}, J=11.7 \mathrm{~Hz}, 3 \mathrm{H}, \mathrm{CH}_{3} \mathrm{O}-\mathrm{N}\right), 3.80(\mathrm{~d}, J=2.6 \mathrm{~Hz}, 3 \mathrm{H}$, $\left.\mathrm{CH}_{3} \mathrm{O}-\mathrm{C}\right), 3.70-3.68(\mathrm{~m}, 1 \mathrm{H}), 3.64-3.43(\mathrm{~m}, 1 \mathrm{H}), 3.35-3.25(\mathrm{~m}, 2 \mathrm{H}), 3.12(\mathrm{t}, J=10.3 \mathrm{~Hz}, 1 \mathrm{H}), 2.37-2.31$ (m, 1H), 1.91 (brs, 1H, OH), 1.47 (d, $J=10.2 \mathrm{~Hz}, 9 \mathrm{H}, \mathrm{Boc}), 1.29-1.19$ (m, 2H, cyclopropyl $\mathrm{CH}_{2}$ ), 1.03 (t, $J=7.7 \mathrm{~Hz}, 2 \mathrm{H}$, cyclopropyl $\left.\mathrm{CH}_{2}\right)$. MS-ESI $(\mathrm{m} / \mathrm{z}): 606.24(\mathrm{M}+\mathrm{H})^{+}$.

7-(3-((S)-2-(tert-Butoxycarbonylamino)-3-(4-hydroxyphenyl)propanamido)-4-(methoxyimino)piperidin-1yl)-1-cyclopropyl-6-fluoro-8-methoxy-4-oxo-1,4-dihydroquinoline-3-carboxylic acid (3i). Obtained from $2 \mathbf{i}$ as a off-white solid (43.9\%), m.p.: $151-153{ }^{\circ} \mathrm{C} .{ }^{1} \mathrm{H}-\mathrm{NMR}\left(400 \mathrm{MHz}, \mathrm{CDCl}_{3}\right) \delta$ (ppm) 14.73 (s, $1 \mathrm{H}, \mathrm{COOH}), 8.82\left(\mathrm{~s}, 1 \mathrm{H}, \mathrm{C}_{2}-\mathrm{H}\right), 7.89-7.84\left(\mathrm{~m}, 1 \mathrm{H}, \mathrm{C}_{5}-\mathrm{H}\right), 7.02(\mathrm{~d}, J=8.1 \mathrm{~Hz}, 2 \mathrm{H}), 6.98-6.76(\mathrm{~m}, 1 \mathrm{H})$, $6.72(\mathrm{~d}, J=8.3 \mathrm{~Hz}, 2 \mathrm{H}), 5.14(\mathrm{~d}, J=35.9 \mathrm{~Hz}, 1 \mathrm{H}), 4.56(\mathrm{~s}, 1 \mathrm{H}), 4.37(\mathrm{~s}, 1 \mathrm{H}), 4.06(\mathrm{~d}, J=12.0 \mathrm{~Hz}, 2 \mathrm{H})$, $3.87\left(\mathrm{~d}, J=10.9 \mathrm{~Hz}, 3 \mathrm{H}, \mathrm{CH}_{3} \mathrm{O}-\mathrm{N}\right), 3.80\left(\mathrm{~d}, J=4.7 \mathrm{~Hz}, 3 \mathrm{H}, \mathrm{CH}_{3} \mathrm{O}-\mathrm{C}\right), 3.76-3.46(\mathrm{~m}, 2 \mathrm{H}), 3.22(\mathrm{~d}$, $J=11.9 \mathrm{~Hz}, 2 \mathrm{H}), 3.03-2.89(\mathrm{~m}, 2 \mathrm{H}), 2.38-2.23(\mathrm{~m}, 1 \mathrm{H}), 1.44$ (s, 9H, Boc), 1.26-1.19 (m, 2H, cyclopropyl $\left.\mathrm{CH}_{2}\right), 1.12-0.94\left(\mathrm{~m}, 2 \mathrm{H}\right.$, cyclopropyl $\left.\mathrm{CH}_{2}\right)$. MS-ESI $(\mathrm{m} / \mathrm{z}): 682.22(\mathrm{M}+\mathrm{H})^{+}$. 
7-(3-((2S,3R)-2-(tert-Butoxycarbonylamino)-3-hydroxybutanamido)-4-(methoxyimino)piperidin-1-yl)1-cyclopropyl-6-fluoro-8-methoxy-4-oxo-1,4-dihydroquinoline-3-carboxylic acid (3j). Obtained from 2j as a white solid (47.7\%), m.p.: $134-136{ }^{\circ} \mathrm{C} .{ }^{1} \mathrm{H}-\mathrm{NMR}\left(400 \mathrm{MHz}, \mathrm{CDCl}_{3}\right) \delta(\mathrm{ppm}) 14.64(\mathrm{~s}, 1 \mathrm{H}$, $\mathrm{COOH}), 8.83\left(\mathrm{~s}, 1 \mathrm{H}, \mathrm{C}_{2}-\mathrm{H}\right), 7.92\left(\mathrm{~d}, J=11.6 \mathrm{~Hz}, 1 \mathrm{H}, \mathrm{C}_{5}-\mathrm{H}\right), 7.33(\mathrm{~s}, 1 \mathrm{H}), 5.42(\mathrm{~s}, 1 \mathrm{H}), 4.74-4.65(\mathrm{~m}$, $1 \mathrm{H}), 4.42-4.33(\mathrm{~m}, 1 \mathrm{H}), 4.17-4.03(\mathrm{~m}, 3 \mathrm{H}), 3.92\left(\mathrm{t}, J=4.3 \mathrm{~Hz}, 3 \mathrm{H}, \mathrm{CH}_{3} \mathrm{O}-\mathrm{N}\right), 3.80$ (s, 3H, $\left.\mathrm{CH}_{3} \mathrm{O}-\mathrm{C}\right)$, $3.61(\mathrm{~s}, 1 \mathrm{H}), 3.29(\mathrm{dd}, J=22.8,13.8 \mathrm{~Hz}, 2 \mathrm{H}), 3.11(\mathrm{t}, J=11.4 \mathrm{~Hz}, 1 \mathrm{H}), 2.43-2.26(\mathrm{~m}, 1 \mathrm{H}), 1.80$ (brs, $1 \mathrm{H}, \mathrm{OH}), 1.46$ (d, $J=11.6 \mathrm{~Hz}, 9 \mathrm{H}, \mathrm{Boc}), 1.26-1.20$ (m, 5H, cyclopropyl $\left.\mathrm{CH}_{2}, \mathrm{CH}_{3} \mathrm{CH}\right), 1.01$ (s, 2H, cyclopropyl $\left.\mathrm{CH}_{2}\right)$. MS-ESI $(\mathrm{m} / \mathrm{z}): 620.21(\mathrm{M}+\mathrm{H})^{+}$.

\subsubsection{General Procedure for the Synthesis of $\mathbf{4 a}-\mathbf{j}$}

To a stirring solution of trifluoroacetic acid $(8.0 \mathrm{~mL})$ was added $\mathbf{3 a}-\mathbf{j}(1.80 \mathrm{mmol})$ in portions over a period of $0.5 \mathrm{~h}$ at $-5-0{ }^{\circ} \mathrm{C}$ and stirred for $3 \mathrm{~h}$ at the same temperature, and then concentrated under reduced pressure. The residue was treated with diethyl ether $(10 \mathrm{~mL})$ and then filtered. The solid was dissolved in methanol $(2 \mathrm{~mL})$ and adjusted to $\mathrm{pH} 7.0$ with the ammonia water, and then extracted with dichloromethane $(30 \mathrm{~mL} \times 3)$. The combined extracts were washed with saturated brine $(10 \mathrm{~mL})$ and dried over anhydrous $\mathrm{Na}_{2} \mathrm{SO}_{4}$, and then concentrated under reduced pressure to provide the title compounds $\mathbf{4 a}-\mathbf{j}$.

7-(3-(2-Aminoacetamido)-4-(methoxyimino)piperidin-1-yl)-1-cyclopropyl-6-fluoro-8-methoxy-4-oxo1,4-dihydroquinoline-3-carboxylic acid (4a). Obtained from 3a as a yellow solid (71.8\%). ${ }^{1} \mathrm{H}-\mathrm{NMR}$ $\left(400 \mathrm{MHz}, \mathrm{CDCl}_{3}\right) \delta(\mathrm{ppm}) 8.83\left(\mathrm{~s}, 1 \mathrm{H}, \mathrm{C}_{2}-\mathrm{H}\right), 8.14(\mathrm{~s}, 1 \mathrm{H}), 7.90\left(\mathrm{~d}, J=8.9 \mathrm{~Hz}, 1 \mathrm{H}, \mathrm{C}_{5}-\mathrm{H}\right), 4.71$ (s, $1 \mathrm{H}), 4.03-3.81(\mathrm{~m}, 6 \mathrm{H}), 3.62(\mathrm{~s}, 1 \mathrm{H}), 3.49-3.12(\mathrm{~m}, 6 \mathrm{H}), 2.41(\mathrm{~s}, 2 \mathrm{H}), 1.25-1.21(\mathrm{~m}, 2 \mathrm{H}$, cyclopropyl $\left.\mathrm{CH}_{2}\right), 1.02\left(\mathrm{~d}, J=17.5 \mathrm{~Hz}, 2 \mathrm{H}\right.$, cyclopropyl $\left.\mathrm{CH}_{2}\right) .{ }^{13} \mathrm{C}-\mathrm{NMR}\left(150 \mathrm{MHz}, \mathrm{DMSO}-d_{6}\right) \delta 176.33,172.48$, 165.59, 156.28, 154.37, 150.61, 146.23, 138.59(d, $J=12.2 \mathrm{~Hz}), 134.06,121.49,106.69,106.46,63.24$, 61.33, 55.74, 49.15, 44.50, 40.73, 24.85, 18.55, 9.03, 8.86. MS-ESI $(\mathrm{m} / z): 476.23(\mathrm{M}+\mathrm{H})^{+}$. HRMS-ESI $(\mathrm{m} / \mathrm{z}): \mathrm{C}_{22} \mathrm{H}_{27} \mathrm{O}_{6} \mathrm{~N}_{5} \mathrm{~F}$, Calcd: 476.193899(M+H) ${ }^{+}$; Found: 476.19389(M+H) ${ }^{+}$.

7-(3-((S)-2-Aminopropanamido)-4-(methoxyimino)piperidin-1-yl)-1-cyclopropyl-6-fluoro-8-methoxy4-oxo-1,4-dihydroquinoline-3-carboxylic acid (4b). Obtained from $\mathbf{3 b}$ as a yellow solid (88.3\%). ${ }^{1} \mathrm{H}-\mathrm{NMR}\left(400 \mathrm{MHz}, \mathrm{CDCl}_{3}\right) \delta(\mathrm{ppm}) 8.81\left(\mathrm{~s}, 1 \mathrm{H}, \mathrm{C}_{2}-\mathrm{H}\right), 8.38-8.00(\mathrm{~m}, 1 \mathrm{H}), 7.87(\mathrm{~d}, J=11.7 \mathrm{~Hz}, 1 \mathrm{H}$, $\left.\mathrm{C}_{5}-\mathrm{H}\right), 4.67(\mathrm{~s}, 1 \mathrm{H}), 4.06(\mathrm{~s}, 2 \mathrm{H}), 3.93\left(\mathrm{~s}, 3 \mathrm{H}, \mathrm{CH}_{3} \mathrm{O}-\mathrm{N}\right), 3.82\left(\mathrm{~s}, 3 \mathrm{H}, \mathrm{CH}_{3} \mathrm{O}-\mathrm{C}\right), 3.57$ (d, J=13.5 Hz, $2 \mathrm{H}), 3.31(\mathrm{~d}, J=11.1 \mathrm{~Hz}, 2 \mathrm{H}), 3.11(\mathrm{~s}, 1 \mathrm{H}), 2.55-2.30(\mathrm{~m}, 1 \mathrm{H}), 1.38-1.33(\mathrm{~m}, 2 \mathrm{H}), 1.26-1.19(\mathrm{~m}, 3 \mathrm{H})$, $1.03(\mathrm{~s}, 2 \mathrm{H}) .{ }^{13} \mathrm{C}-\mathrm{NMR}\left(100 \mathrm{MHz}, \mathrm{CDCl}_{3}\right) \delta 177.15,175.65,166.78,157.70,153.47,150.12,146.20$, $139.02(\mathrm{~d}, J=12 \mathrm{~Hz}), 133.91,122.77,108.40,107.96,62.84,62.06,56.65,51.00,50.64,50.24,40.70$, 25.67, 21.77, 9.86, 9.53. MS-ESI $(\mathrm{m} / \mathrm{z}): 490.25(\mathrm{M}+\mathrm{H})^{+}$.HRMS-ESI $(\mathrm{m} / z): \mathrm{C}_{23} \mathrm{H}_{29} \mathrm{O}_{6} \mathrm{~N}_{5} \mathrm{~F}$, Calcd: $490.20964(\mathrm{M}+\mathrm{H})^{+}$; Found: $490.20968(\mathrm{M}+\mathrm{H})^{+}$.

7-(3-((S)-2-Amino-3-methylbutanamido)-4-(methoxyimino)piperidin-1-yl)-1-cyclopropyl-6-fluoro-8methoxy-4-oxo-1,4-dihydroquinoline-3-carboxylic acid (4c). Obtained from 3c as a light yellow solid (70.0\%). ${ }^{1} \mathrm{H}-\mathrm{NMR}\left(400 \mathrm{MHz}, \mathrm{DMSO}-d_{6}\right) \delta(\mathrm{ppm}) 8.70\left(\mathrm{~s}, 1 \mathrm{H}, \mathrm{C}_{2}-\mathrm{H}\right), 8.29-8.24(\mathrm{~m}, 1 \mathrm{H}), 7.77(\mathrm{~d}$, $\left.J=10.6 \mathrm{~Hz}, 1 \mathrm{H}, \mathrm{C}_{5}-\mathrm{H}\right), 4.59-4.55(\mathrm{~m}, 1 \mathrm{H}), 4.16(\mathrm{~s}, 1 \mathrm{H}), 3.83\left(\mathrm{~s}, 3 \mathrm{H}, \mathrm{CH}_{3} \mathrm{O}-\mathrm{N}\right), 3.76(\mathrm{~d}, J=5.2 \mathrm{~Hz}$, $\left.3 \mathrm{H}, \mathrm{CH}_{3} \mathrm{O}-\mathrm{N}\right), 3.73-3.58(\mathrm{~m}, 1 \mathrm{H}), 3.56-3.41(\mathrm{~m}, 1 \mathrm{H}), 3.28-3.16(\mathrm{~m}, 1 \mathrm{H}), 3.03(\mathrm{~d}, J=3.5 \mathrm{~Hz}, 2 \mathrm{H})$, 
2.60-2.50 (m, 1H), 1.98-1.94 (m, 1H), 1.91-1.72 (m, 1H), 1.21-0.91 (m, 4H, $2 \times$ cyclopropyl $\left.\mathrm{CH}_{2}\right)$, $0.87(\mathrm{~d}, J=6.8 \mathrm{~Hz}, 1 \mathrm{H}), 0.80(\mathrm{~d}, J=6.8 \mathrm{~Hz}, 2 \mathrm{H}), 0.74-0.70(\mathrm{~m}, 3 \mathrm{H}) .{ }^{13} \mathrm{C}-\mathrm{NMR}\left(100 \mathrm{MHz}, \mathrm{DMSO}-d_{6}\right)$ $\delta 176.30,174.16,165.61,156.74,154.45,150.62,146.08(\mathrm{~d}, J=18 \mathrm{~Hz}), 138.60,134.04,121.33$, 106.65, 106.42, 63.11, 61.27, 59.66, 55.47, 49.25, 40.71, 30.30, 24.76, 19.29, 16.65, 16.53, 8.98, 8.88. MS-ESI $(\mathrm{m} / \mathrm{z}): 518.35(\mathrm{M}+\mathrm{H})^{+}$.HRMS-ESI $(\mathrm{m} / \mathrm{z}): \mathrm{C}_{25} \mathrm{H}_{33} \mathrm{O}_{6} \mathrm{~N}_{5} \mathrm{~F}$, Calcd: $518.24094(\mathrm{M}+\mathrm{H})^{+}$; Found: $518.24080(\mathrm{M}+\mathrm{H})^{+}$.

7-(3-((S)-2-Amino-4-methylpentanamido)-4-(methoxyimino)piperidin-1-yl)-1-cyclopropyl-6-fluoro-8methoxy-4-oxo-1,4-dihydroquinoline-3-carboxylic acid (4d). Obtained from 3d as a yellow solid (71.5\%). ${ }^{1} \mathrm{H}-\mathrm{NMR}\left(400 \mathrm{MHz}, \mathrm{DMSO}-d_{6}\right) \delta(\mathrm{ppm}) 8.71\left(\mathrm{~s}, 1 \mathrm{H}, \mathrm{C}_{2}-\mathrm{H}\right), 8.46-8.23(\mathrm{~m}, 1 \mathrm{H}), 7.77(\mathrm{~d}$, $\left.J=10.6 \mathrm{~Hz}, 1 \mathrm{H}, \mathrm{C}_{5}-\mathrm{H}\right), 4.55-4.49(\mathrm{~m}, 1 \mathrm{H}), 4.17(\mathrm{~s}, 1 \mathrm{H}), 3.83\left(\mathrm{~s}, 3 \mathrm{H}, \mathrm{CH}_{3} \mathrm{O}-\mathrm{N}\right), 3.76(\mathrm{~d}, J=8.3 \mathrm{~Hz}$, $\left.3 \mathrm{H}, \mathrm{CH}_{3} \mathrm{O}-\mathrm{C}\right), 3.69(\mathrm{~s}, 1 \mathrm{H}), 3.48-3.21(\mathrm{~m}, 3 \mathrm{H}), 3.03$ (t, $\left.J=16.1 \mathrm{~Hz}, 1 \mathrm{H}\right), 2.64(\mathrm{~d}, J=6.4 \mathrm{~Hz}, 1 \mathrm{H})$, $1.72(\mathrm{~s}, 1 \mathrm{H}), 1.56(\mathrm{~s}, 1 \mathrm{H}), 1.41(\mathrm{~s}, 1 \mathrm{H}), 1.35-0.96(\mathrm{~m}, 5 \mathrm{H}), 0.86-0.81(\mathrm{~m}, 3 \mathrm{H}), 0.77-0.73(\mathrm{~m}, 3 \mathrm{H})$. ${ }^{13} \mathrm{C}-\mathrm{NMR}\left(100 \mathrm{MHz}, \mathrm{DMSO}-d_{6}\right) \delta 176.31,175.04,165.60,156.72,154.44,150.60,146.02(\mathrm{~d}, J=33 \mathrm{~Hz})$, 138.66, 134.03, 121.43, 106.65, 106.42, 63.13, 61.27, 55.51, 52.99, 49.16, 44.07, 40.70, 24.87, 23.97, 23.12, 23.03, 21.85, 8.97, 8.93. MS-ESI $(\mathrm{m} / \mathrm{z}): 532.40(\mathrm{M}+\mathrm{H})^{+}$. HRMS-ESI $(\mathrm{m} / \mathrm{z}): \mathrm{C}_{26} \mathrm{H}_{35} \mathrm{O}_{6} \mathrm{~N}_{5} \mathrm{~F}$, Calcd: $532.25659(\mathrm{M}+\mathrm{H})^{+}$; Found: $532.25671(\mathrm{M}+\mathrm{H})^{+}$.

7-(4-(methoxyimino)-3-((S)-pyrrolidine-2-carboxamido)piperidin-1-yl)-1-Cyclopropyl-6-fluoro-8methoxy-4-oxo-1,4-dihydroquinoline-3-carboxylic acid (4e). Obtained from $\mathbf{3 e}$ as a yellow solid (66.8\%). ${ }^{1} \mathrm{H}-\mathrm{NMR}\left(400 \mathrm{MHz}, \mathrm{CDCl}_{3}\right) \delta(\mathrm{ppm}) 8.81\left(\mathrm{~s}, 1 \mathrm{H}, \mathrm{C}_{2}-\mathrm{H}\right), 8.45-8.39(\mathrm{~m}, 1 \mathrm{H}), 7.87(\mathrm{~d}, J=11.7 \mathrm{~Hz}$, $\left.1 \mathrm{H}, \mathrm{C}_{5}-\mathrm{H}\right), 4.67(\mathrm{~s}, 1 \mathrm{H}), 4.04-3.98(\mathrm{~m}, 2 \mathrm{H}), 3.92\left(\mathrm{~d}, J=7.8 \mathrm{~Hz}, 3 \mathrm{H}, \mathrm{CH}_{3} \mathrm{O}-\mathrm{N}\right), 3.81(\mathrm{~s}, 4 \mathrm{H}), 3.57(\mathrm{~d}$, $J=12.5 \mathrm{~Hz}, 1 \mathrm{H}), 3.31-3.27(\mathrm{~m}, 2 \mathrm{H}), 3.15(\mathrm{t}, J=10.7 \mathrm{~Hz}, 1 \mathrm{H}), 3.05-2.93(\mathrm{~m}, 2 \mathrm{H}), 2.51-2.31(\mathrm{~m}, 1 \mathrm{H})$, 2.15-2.10 (m, 1H), 2.00-1.95 (m, 1H), 1.88-1.56 (m, 2H), 1.24-1.19 (m, 2H, cyclopropyl $\left.\mathrm{CH}_{2}\right), 1.02$ (s, 2H). ${ }^{13} \mathrm{C}-\mathrm{NMR}\left(100 \mathrm{MHz}, \mathrm{CDCl}_{3}\right) \delta 177.12,175.17,166.76,157.61,153.61,150.11,146.10$, 138.97 (d, $J=12 \mathrm{~Hz}), 133.91,122.64,108.41,107.97,62.81,61.96,60.82,56.59,50.52,49.88,47.37$, 40.68, 30.92, 26.21, 25.61, 9.86, 9.53. MS-ESI $(\mathrm{m} / \mathrm{z}): 516.36(\mathrm{M}+\mathrm{H})^{+} \cdot$ HRMS-ESI $(\mathrm{m} / \mathrm{z}): \mathrm{C}_{25} \mathrm{H}_{31} \mathrm{O}_{6} \mathrm{~N}_{5} \mathrm{~F}$, Calcd: $516.22529(\mathrm{M}+\mathrm{H})^{+}$; Found: $516.22545(\mathrm{M}+\mathrm{H})^{+}$.

7-(3-((2S,3S)-2-Amino-3-methylpentanamido)-4-(methoxyimino)piperidin-1-yl)-1-cyclopropyl-6fluoro-8-methoxy-4-oxo-1,4-dihydroquinoline-3-carboxylic acid (4f). Obtained from $\mathbf{3 f}$ as a yellow solid (88.3\%). ${ }^{1} \mathrm{H}-\mathrm{NMR}\left(400 \mathrm{MHz}, \mathrm{CDCl}_{3}\right) \delta(\mathrm{ppm}) 8.73\left(\mathrm{~d}, J=3.5 \mathrm{~Hz}, 1 \mathrm{H}, \mathrm{C}_{2}-\mathrm{H}\right), 7.91-7.57(\mathrm{~m}, 1 \mathrm{H}$, $\left.\mathrm{C}_{5}-\mathrm{H}\right), 7.46(\mathrm{~s}, 1 \mathrm{H}), 4.71(\mathrm{~s}, 1 \mathrm{H}), 4.13-4.04(\mathrm{~m}, 3 \mathrm{H}), 3.90\left(\mathrm{~d}, J=6.9 \mathrm{~Hz}, 3 \mathrm{H}, \mathrm{CH}_{3} \mathrm{O}-\mathrm{N}\right), 3.79(\mathrm{~s}, 3 \mathrm{H}$, $\left.\mathrm{CH}_{3} \mathrm{O}-\mathrm{C}\right), 3.57-3.47(\mathrm{~m}, 1 \mathrm{H}), 3.34-3.19(\mathrm{~m}, 2 \mathrm{H}), 2.41(\mathrm{~s}, 1 \mathrm{H}), 2.13(\mathrm{~s}, 1 \mathrm{H}), 1.63-1.58(\mathrm{~m}, 1 \mathrm{H}), 1.22$ $(\mathrm{d}, J=6.4 \mathrm{~Hz}, 4 \mathrm{H}), 1.06-0.97(\mathrm{~m}, 6 \mathrm{H}), 0.92(\mathrm{~s}, 2 \mathrm{H}) .{ }^{13} \mathrm{C}-\mathrm{NMR}\left(100 \mathrm{MHz}, \mathrm{CDCl}_{3}\right) \delta 176.74,175.34$, $166.81,154.83,153.05,150.25,145.93(\mathrm{~d}, J=12 \mathrm{~Hz}), 138.91,134.05,122.08,107.94,107.61,63.12$, $62.15,58.19,55.96,50.94,50.48,40.87,37.01,25.38,14.86,11.75,9.76$. MS-ESI $(\mathrm{m} / \mathrm{z}): 532.33$ $(\mathrm{M}+\mathrm{H})^{+}$. HRMS-ESI $(\mathrm{m} / \mathrm{z}): \mathrm{C}_{26} \mathrm{H}_{35} \mathrm{O}_{6} \mathrm{~N}_{5} \mathrm{~F}$, Calcd: $532.25659(\mathrm{M}+\mathrm{H})^{+}$; Found: $532.25653(\mathrm{M}+\mathrm{H})^{+}$.

7-(3-((S)-2-Amino-3-phenylpropanamido)-4-(methoxyimino)piperidin-1-yl)-1-cyclopropyl-6-fluoro-8methoxy-4-oxo-1,4-dihydroquinoline-3-carboxylic acid (4g). Obtained from $\mathbf{3 g}$ as a yellow solid (82.4\%). ${ }^{1} \mathrm{H}-\mathrm{NMR}\left(400 \mathrm{MHz}, \mathrm{DMSO}-d_{6}\right) \delta(\mathrm{ppm}) 8.72\left(\mathrm{~s}, 1 \mathrm{H}, \mathrm{C}_{2}-\mathrm{H}\right), 8.28(\mathrm{~d}, J=4.6 \mathrm{~Hz}, 1 \mathrm{H})$, 7.90-7.70 (m, 1H, C $5-\mathrm{H}), 7.40-6.90$ (m, 5H, ph-H), 4.54 (s, 1H), 4.17 (s, 1H), 3.83 (d, J=8.0 Hz, 3H, 
$\left.\mathrm{CH}_{3} \mathrm{O}-\mathrm{N}\right), 3.76-3.66(\mathrm{~m}, 4 \mathrm{H}), 3.47(\mathrm{~d}, J=5.7 \mathrm{~Hz}, 2 \mathrm{H}), 3.31(\mathrm{~s}, 2 \mathrm{H}), 3.23-3.07(\mathrm{~m}, 1 \mathrm{H}), 2.96-2.91(\mathrm{~m}$, 2H), 2.74-2.51 (m, 1H), 1.26-0.83 (m, 4H, $2 \times$ cyclopropyl $\left.\mathrm{CH}_{2}\right) .{ }^{13} \mathrm{C}-\mathrm{NMR}\left(100 \mathrm{MHz}, \mathrm{DMSO}-d_{6}\right) \delta$ 176.29, 173.76, 165.66, 156.71, 154.33, 150.64, 146.24, 138.35(d, $J=29 \mathrm{~Hz}), 134.00,129.24,128.02$, 126.01, 121.57, 106.69, 106.46, 63.14, 61.30, 56.00, 55.68, 49.25, 40.68, 40.45, 24.70, 16.37, 8.95. MS-ESI $(\mathrm{m} / \mathrm{z}): 566.40(\mathrm{M}+\mathrm{H})^{+}$. HRMS-ESI $(\mathrm{m} / \mathrm{z}): \mathrm{C}_{29} \mathrm{H}_{33} \mathrm{O}_{6} \mathrm{~N}_{5} \mathrm{~F}$, Calcd: $566.24094(\mathrm{M}+\mathrm{H})^{+}$; Found: $566.24108(\mathrm{M}+\mathrm{H})^{+}$.

7-(3-((S)-2-Amino-3-hydroxypropanamido)-4-(methoxyimino)piperidin-1-yl)-1-cyclopropyl-6-fluoro8-methoxy-4-oxo-1,4-dihydroquinoline-3-carboxylic acid $(\mathbf{4 h})$. Obtained from $\mathbf{3 h}$ as a yellow solid (66.2\%). ${ }^{1} \mathrm{H}-\mathrm{NMR}\left(400 \mathrm{MHz}, \mathrm{DMSO}-d_{6}\right) \delta(\mathrm{ppm}) 8.71\left(\mathrm{~s}, 1 \mathrm{H}, \mathrm{C}_{2}-\mathrm{H}\right), 8.36(\mathrm{~d}, J=5.1 \mathrm{~Hz}, 1 \mathrm{H}), 7.78(\mathrm{~d}$, $\left.J=8.0 \mathrm{~Hz}, 1 \mathrm{H}, \mathrm{C}_{5}-\mathrm{H}\right), 4.76(\mathrm{~s}, 1 \mathrm{H}), 4.56-4.52(\mathrm{~m}, 1 \mathrm{H}), 4.17(\mathrm{~s}, 1 \mathrm{H}), 3.84\left(\mathrm{~d}, J=0.9 \mathrm{~Hz}, 3 \mathrm{H}, \mathrm{CH}_{3} \mathrm{O}-\mathrm{N}\right)$, 3.81-3.70 (m, 4H), 3.60-3.35 (m, 4H), 3.26-3.25 (m, 2H), 3.11-2.96 (m, 1H), 2.56-2.51 (m, 1H), $1.13-0.96\left(\mathrm{~m}, 4 \mathrm{H}, 2 \times\right.$ cyclopropyl $\left.\mathrm{CH}_{2}\right) .{ }^{13} \mathrm{C}-\mathrm{NMR}\left(100 \mathrm{MHz}, \mathrm{DMSO}-d_{6}\right) \delta 176.36,172.77,165.60$, 156.82, 154.37, 150.61, 146.29 (d, $J=11 \mathrm{~Hz}), 138.67,134.05,121.49,106.69,106.42,64.90,63.18$, $61.34,56.76,55.65,49.40,40.75,24.85,15.16,8.92$. MS-ESI $(\mathrm{m} / z): 506.28(\mathrm{M}+\mathrm{H})^{+}$.HRMS-ESI $(\mathrm{m} / \mathrm{z})$ : $\mathrm{C}_{23} \mathrm{H}_{29} \mathrm{O}_{7} \mathrm{~N}_{5} \mathrm{~F}$, Calcd: $506.20455(\mathrm{M}+\mathrm{H})^{+}$; Found: $506.20465(\mathrm{M}+\mathrm{H})^{+}$.

7-(3-((S)-2-Amino-3-(4-hydroxyphenyl)propanamido)-4-(methoxyimino)piperidin-1-yl)-1-cyclopropyl6-fluoro-8-methoxy-4-oxo-1,4-dihydroquinoline-3-carboxylic acid (4i). Obtained from 3i as a light yellow solid (83.4\%). ${ }^{1} \mathrm{H}-\mathrm{NMR}\left(400 \mathrm{MHz}, \mathrm{DMSO}-d_{6}\right) \delta(\mathrm{ppm}) 9.11(\mathrm{~d}, J=15.9 \mathrm{~Hz}, 1 \mathrm{H}), 8.71(\mathrm{~d}$, $\left.J=4.5 \mathrm{~Hz}, 1 \mathrm{H}, \mathrm{C}_{2}-\mathrm{H}\right), 8.23(\mathrm{t}, J=8.0 \mathrm{~Hz}, 1 \mathrm{H}), 7.79\left(\mathrm{dd}, J=11.8,6.1 \mathrm{~Hz}, 1 \mathrm{H}, \mathrm{C}_{5}-\mathrm{H}\right), 6.95(\mathrm{t}, J=7.6 \mathrm{~Hz}$, 2H), 6.59 (dd, $J=14.6,8.3 \mathrm{~Hz}, 2 \mathrm{H}), 4.53(\mathrm{~s}, 1 \mathrm{H}), 4.18(\mathrm{dd}, J=6.8,3.8 \mathrm{~Hz}, 1 \mathrm{H}), 3.83$ (d, $J=7.8 \mathrm{~Hz}$, $\left.3 \mathrm{H}, \mathrm{CH}_{3} \mathrm{O}-\mathrm{N}\right), 3.74\left(\mathrm{~d}, J=10.8 \mathrm{~Hz}, 3 \mathrm{H}, \mathrm{CH}_{3} \mathrm{O}-\mathrm{C}\right), 3.67(\mathrm{~d}, J=7.8 \mathrm{~Hz}, 1 \mathrm{H}), 3.54-3.44(\mathrm{~m}, 1 \mathrm{H})$, 3.42-3.34 (m, 2H), 3.26-3.16 (m, 1H), 3.15-2.89 (m, 2H), 2.79 (d, $J=11.0 \mathrm{~Hz}, 1 \mathrm{H}), 2.65-2.53(\mathrm{~m}$, 1H), $1.16-1.01\left(\mathrm{~m}, 4 \mathrm{H}, 2 \times\right.$ cyclopropyl $\left.\mathrm{CH}_{2}\right) .{ }^{13} \mathrm{C}-\mathrm{NMR}\left(100 \mathrm{MHz}, \mathrm{DMSO}-d_{6}\right) \delta 176.40,173.94$, $165.64,156.74,155.78,154.30,150.63,146.26,138.72(\mathrm{~d}, J=38 \mathrm{~Hz}), 134.07,130.27,130.09,128.33$, 127.95, 121.53, 114.88, 106.69, 106.48, 63.15, 61.31, 56.18, 55.80, 49.71, 49.24, 40.75, 24.87, 15.15, 9.06, 8.96. MS-ESI $(m / z): 582.30(\mathrm{M}+\mathrm{H})^{+}$.HRMS-ESI $(m / z): \mathrm{C}_{29} \mathrm{H}_{33} \mathrm{O}_{7} \mathrm{~N}_{5} \mathrm{~F}$, Calcd: $582.23585(\mathrm{M}+\mathrm{H})^{+}$; Found: $582.23593(\mathrm{M}+\mathrm{H})^{+}$.

7-(3-((2S,3R)-2-Amino-3-hydroxybutanamido)-4-(methoxyimino)piperidin-1-yl)-1-cyclopropyl-6-

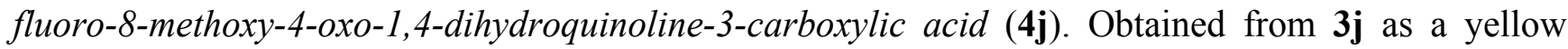
solid (73.3\%). ${ }^{1} \mathrm{H}-\mathrm{NMR}\left(400 \mathrm{MHz}, \mathrm{DMSO}-d_{6}\right) \delta(\mathrm{ppm}) 8.71\left(\mathrm{~s}, 1 \mathrm{H}, \mathrm{C}_{2}-\mathrm{H}\right), 8.38(\mathrm{~d}, J=7.0 \mathrm{~Hz}, 1 \mathrm{H})$, $7.78\left(\mathrm{dd}, J=11.8,3.1 \mathrm{~Hz}, 1 \mathrm{H}, \mathrm{C}_{5}-\mathrm{H}\right), 4.63-4.50(\mathrm{~m}, 1 \mathrm{H}), 4.22-4.10(\mathrm{~m}, 1 \mathrm{H}), 3.91-3.80(\mathrm{~m}, 4 \mathrm{H})$, $3.79-3.59(\mathrm{~m}, 4 \mathrm{H}), 3.51-3.47(\mathrm{~m}, 1 \mathrm{H}), 3.31-3.12(\mathrm{~m}, 2 \mathrm{H}), 3.10-3.02(\mathrm{~m}, 1 \mathrm{H}), 2.99(\mathrm{~d}, J=4.1 \mathrm{~Hz}, 1 \mathrm{H})$, 2.60-2.51 (m, 1H), 1.29-1.23 (m, 1H), 1.13-1.11 (m, 2H), 1.04-0.97 (m, 3H), 0.91-0.73 (m, 1H). ${ }^{13} \mathrm{C}-\mathrm{NMR}(100 \mathrm{MHz}, \mathrm{DMSO}-d 6) \delta$ 176.34, 173.04, 165.60, 156.84, 154.39, 150.61, 146.10, $138.56(\mathrm{~d}$, $J=38 \mathrm{~Hz}), 134.06,122.24,106.66,106.42,67.14,63.17,61.32,60.19,55.60,49.47,39.94,24.85$, 20.08, 19.94, 8.94. MS-ESI $(m / z): 520.28(\mathrm{M}+\mathrm{H})^{+}$. HRMS-ESI $(\mathrm{m} / z): \mathrm{C}_{24} \mathrm{H}_{31} \mathrm{O}_{7} \mathrm{~N}_{5} \mathrm{~F}$, Calcd: 520.22020 $(\mathrm{M}+\mathrm{H})^{+}$; Found: $520.22083(\mathrm{M}+\mathrm{H})^{+}$. 


\subsubsection{General Procedure for the Synthesis of $\mathbf{3 k}-\mathbf{l}$}

A mixture of $\mathbf{4 a}-\mathbf{b}(0.82 \mathrm{mmol}), \mathbf{2 a}-\mathbf{b}(0.82 \mathrm{mmol})$, dicyclohexylcarbodiimide $(0.17 \mathrm{~g}, 0.82 \mathrm{mmol})$ and dry dichloromethane $(20 \mathrm{~mL})$ was stirred at room temperature for $4 \mathrm{~h}$, filtered and concentrated under reduced pressure. The solid was treated with diethyl ether $(3 \mathrm{~mL})$ and filtered. The solid was purified by column chromatography (silica gel) eluted with dichloromethane and methanol $(\mathrm{v}: \mathrm{v}=60: 1)$ to afford the title compounds $3 \mathbf{k}-\mathbf{l}$.

7-(3-(2-(2-(tert-Butoxycarbonylamino)acetamido)acetamido)-4-(methoxyimino)piperidin-1-yl)-1cyclopropyl-6-fluoro-8-methoxy-4-oxo-1,4-dihydroquinoline-3-carboxylic acid (3k). Obtained from 4a and 2a as a light yellow solid (30.8\%), m.p.: 122-124 ${ }^{\circ} \mathrm{C} .{ }^{1} \mathrm{H}-\mathrm{NMR}\left(400 \mathrm{MHz}, \mathrm{CDCl}_{3}\right) \delta(\mathrm{ppm}) 14.71$ (s, 1H, COOH), 8.83 (s, 1H, C $2-\mathrm{H}), 7.90$ (d, $\left.J=11.6 \mathrm{~Hz}, 1 \mathrm{H}, \mathrm{C}_{5}-\mathrm{H}\right), 6.97$ (d, J=5.6 Hz, 1H), 6.75 (s, $1 \mathrm{H}), 5.07(\mathrm{~s}, 1 \mathrm{H}), 4.72-4.56(\mathrm{~m}, 1 \mathrm{H}), 4.11(\mathrm{~d}, J=7.0 \mathrm{~Hz}, 1 \mathrm{H}), 4.06-3.95(\mathrm{~m}, 3 \mathrm{H}), 3.93\left(\mathrm{~s}, 3 \mathrm{H}, \mathrm{CH}_{3} \mathrm{O}-\right.$ $\mathrm{N}), 3.86(\mathrm{~d}, J=3.7 \mathrm{~Hz}, 2 \mathrm{H}), 3.78\left(\mathrm{~s}, 3 \mathrm{H}, \mathrm{CH}_{3} \mathrm{O}-\mathrm{C}\right), 3.61(\mathrm{~d}, J=10.0 \mathrm{~Hz}, 1 \mathrm{H}), 3.34-3.24(\mathrm{~m}, 2 \mathrm{H})$, $3.07(\mathrm{t}, J=10.5 \mathrm{~Hz}, 1 \mathrm{H}), 2.42-2.26(\mathrm{~m}, 1 \mathrm{H}), 1.45(\mathrm{~s}, 9 \mathrm{H}, \mathrm{Boc}), 1.26-1.21\left(\mathrm{~m}, 2 \mathrm{H}\right.$, cyclopropyl $\left.\mathrm{CH}_{2}\right)$, $1.07-0.98\left(\mathrm{~m}, 2 \mathrm{H}\right.$, cyclopropyl $\left.\mathrm{CH}_{2}\right)$. MS-ESI $(\mathrm{m} / \mathrm{z}): 633.17(\mathrm{M}+\mathrm{H})^{+}$.

\section{7-(3-((S)-2-((S)-2-(tert-Butoxycarbonylamino)propanamido)propanamido)-4-(methoxyimino)piperidin-1-}

yl)-1-cyclopropyl-6-fluoro-8-methoxy-4-oxo-1,4-dihydroquinoline-3-carboxylic acid (31). Obtained from $4 \mathbf{b}$ and $2 \mathbf{b}$ as a yellow solid (71.5\%), m.p.: $126-128{ }^{\circ} \mathrm{C} .{ }^{1} \mathrm{H}-\mathrm{NMR}\left(400 \mathrm{MHz}, \mathrm{CDCl}_{3}\right) \delta(\mathrm{ppm})$ $14.68(\mathrm{~s}, 1 \mathrm{H}, \mathrm{COOH}), 8.83\left(\mathrm{~s}, 1 \mathrm{H}, \mathrm{C}_{2}-\mathrm{H}\right), 7.90$ (d, $\left.J=11.6 \mathrm{~Hz}, 1 \mathrm{H}, \mathrm{C}_{5}-\mathrm{H}\right), 7.18-6.92(\mathrm{~m}, 1 \mathrm{H}), 6.71(\mathrm{~d}$, $J=20.8 \mathrm{~Hz}, 1 \mathrm{H}), 4.93(\mathrm{~s}, 1 \mathrm{H}), 4.70-4.58(\mathrm{~m}, 1 \mathrm{H}), 4.53-4.48(\mathrm{~m}, 1 \mathrm{H}), 4.16(\mathrm{~s}, 1 \mathrm{H}), 4.16-4.03(\mathrm{~m}, 2 \mathrm{H})$, $3.92\left(\mathrm{~d}, J=4.0 \mathrm{~Hz}, 3 \mathrm{H}, \mathrm{CH}_{3} \mathrm{O}-\mathrm{N}\right), 3.79$ (d, $\left.J=1.5 \mathrm{~Hz}, 3 \mathrm{H}, \mathrm{CH}_{3} \mathrm{O}-\mathrm{C}\right), 3.59$ (s, $\left.1 \mathrm{H}\right), 3.40-3.18(\mathrm{~m}, 2 \mathrm{H})$, 3.14-2.97 (m, 1H), 2.34-2.32 (m, 1H), 1.63-1.29 (m, 15H, Boc, $\left.2 \times \mathrm{CH}_{3}\right), 1.28-1.16(\mathrm{~m}, 2 \mathrm{H}$, cyclopropyl $\left.\mathrm{CH}_{2}\right), 1.09-0.87\left(\mathrm{~m}, 2 \mathrm{H}\right.$, cyclopropyl $\left.\mathrm{CH}_{2}\right)$. MS-ESI $(\mathrm{m} / \mathrm{z}): 661.33(\mathrm{M}+\mathrm{H})^{+}$.

\subsubsection{General Procedure for the Synthesis of $4 \mathbf{k}-\mathbf{l}$}

To a stirring solution of trifluoroacetic acid $(3.0 \mathrm{~mL})$ was added $\mathbf{3 k}-\mathbf{l}(0.47 \mathrm{mmol})$ in portions over a period of $0.5 \mathrm{~h}$ at $-5-0{ }^{\circ} \mathrm{C}$, stirred for $2 \mathrm{~h}$ at the same temperature and then concentrated under reduced pressure. The residue was treated with diethyl ether $(7 \mathrm{~mL})$ and filtered. The solid was dissolved in methanol $(1 \mathrm{~mL})$ and adjusted to $\mathrm{pH} 7.0$ with the ammonia water, and then extracted with dichloromethane $(50 \mathrm{~mL} \times 3)$. The combined extracts were washed with saturated brine $(10 \mathrm{~mL})$, dried over anhydrous $\mathrm{Na}_{2} \mathrm{SO}_{4}$, and concentrated under reduced pressure to provide the title compounds $\mathbf{4 k}-\mathbf{l}$.

\section{7-(3-(2-(2-Aminoacetamido)acetamido)-4-(methoxyimino)piperidin-1-yl)-1-cyclopropyl-6-fluoro-8-}

methoxy-4-oxo-1,4-dihydroquinoline-3-carboxylic acid (4k). Obtained from $\mathbf{3 k}$ as a yellow solid (73.3\%). ${ }^{1} \mathrm{H}-\mathrm{NMR}\left(400 \mathrm{MHz}, \mathrm{CDCl}_{3}\right) \delta(\mathrm{ppm}) 8.81\left(\mathrm{~s}, 1 \mathrm{H}, \mathrm{C}_{2}-\mathrm{H}\right), 7.98-7.80(\mathrm{~m}, 2 \mathrm{H}), 7.11(\mathrm{~d}, J=5.9 \mathrm{~Hz}$, $1 \mathrm{H}), 4.75-4.56(\mathrm{~m}, 1 \mathrm{H}), 4.14-4.10(\mathrm{~m}, 1 \mathrm{H}), 4.07-4.00(\mathrm{~m}, 2 \mathrm{H}), 3.97(\mathrm{~d}, J=5.6 \mathrm{~Hz}, 1 \mathrm{H}), 3.92(\mathrm{~s}, 3 \mathrm{H}$, $\left.\mathrm{CH}_{3} \mathrm{O}-\mathrm{N}\right), 3.79\left(\mathrm{~s}, 3 \mathrm{H}, \mathrm{CH}_{3} \mathrm{O}-\mathrm{C}\right), 3.60(\mathrm{~d}, J=8.0 \mathrm{~Hz}, 1 \mathrm{H}), 3.52-3.44(\mathrm{~m}, 1 \mathrm{H}), 3.44(\mathrm{~s}, 1 \mathrm{H}), 3.35-3.19$ $(\mathrm{m}, 2 \mathrm{H}), 3.09$ (t, $J=10.7 \mathrm{~Hz}, 1 \mathrm{H}), 2.46-2.22(\mathrm{~m}, 1 \mathrm{H}), 1.27-1.18\left(\mathrm{~m}, 2 \mathrm{H}\right.$, cyclopropyl $\left.\mathrm{CH}_{2}\right), 1.13-0.95$ (m, 2H, cyclopropyl $\left.\mathrm{CH}_{2}\right) .{ }^{13} \mathrm{C}-\mathrm{NMR}\left(100 \mathrm{MHz} \mathrm{CDCl}_{3}\right) \delta 177.07,173.52,168.75,166.74,157.52$, 152.99, 150.12, 146.05, 138.85(d, $J=12 \mathrm{~Hz}), 133.94,122.74,108.33,107.96,62.95,62.11,56.43$, 
50.57, 50.15, 44.79, 42.87, 40.70, 25.66, 9.86, 9.53. MS-ESI $(\mathrm{m} / \mathrm{z}): 533.32(\mathrm{M}+\mathrm{H})^{+}$.HRMS-ESI $(\mathrm{m} / \mathrm{z})$ : $\mathrm{C}_{24} \mathrm{H}_{30} \mathrm{O}_{7} \mathrm{~N}_{6} \mathrm{~F}$, Calcd: $533.21545(\mathrm{M}+\mathrm{H})^{+}$; Found: $533.21660(\mathrm{M}+\mathrm{H})^{+}$.

7-(3-((S)-2-((S)-2-Aminopropanamido)propanamido)-4-(methoxyimino)piperidin-1-yl)-1-cyclopropyl6-fluoro-8-methoxy-4-oxo-1,4-dihydroquinoline-3-carboxylic acid (41). Obtained from 31 as a yellow solid (34.7\%). ${ }^{1} \mathrm{H}-\mathrm{NMR}\left(400 \mathrm{MHz}, \mathrm{CDCl}_{3}\right) \delta(\mathrm{ppm}) 8.81\left(\mathrm{~s}, 1 \mathrm{H}, \mathrm{C}_{2}-\mathrm{H}\right), 7.86(\mathrm{dd}, J=11.6,6.2 \mathrm{~Hz}, 1 \mathrm{H}$, $\left.\mathrm{C}_{5}-\mathrm{H}\right), 7.75(\mathrm{t}, J=7.8 \mathrm{~Hz}, 1 \mathrm{H}), 7.28-7.10(\mathrm{~m}, 1 \mathrm{H}), 4.66-4.62(\mathrm{~m}, 1 \mathrm{H}), 4.56-4.47(\mathrm{~m}, 1 \mathrm{H}), 4.13-4.00$ $(\mathrm{m}, 2 \mathrm{H}), 3.92\left(\mathrm{~d}, J=4.6 \mathrm{~Hz}, 3 \mathrm{H}, \mathrm{CH}_{3} \mathrm{O}-\mathrm{N}\right), 3.80\left(\mathrm{~s}, 3 \mathrm{H}, \mathrm{CH}_{3} \mathrm{O}-\mathrm{C}\right), 3.68-3.44(\mathrm{~m}, 2 \mathrm{H}), 3.32-3.25$ (m, $2 \mathrm{H}), 3.08(\mathrm{t}, J=10.4 \mathrm{~Hz}, 1 \mathrm{H}), 2.34(\mathrm{t}, J=12.7 \mathrm{~Hz}, 1 \mathrm{H}), 1.44-1.33(\mathrm{~m}, 5 \mathrm{H}), 1.26-1.21(\mathrm{~m}, 3 \mathrm{H}), 1.03(\mathrm{~s}$, 2H). ${ }^{13} \mathrm{C}-\mathrm{NMR}\left(100 \mathrm{MHz}, \mathrm{CDCl}_{3}\right) \delta 177.11,175.83,172.12,166.76,159.46,153.12,150.11$, 146.14(d, $J=12 \mathrm{~Hz}), 138.80,133.90,122.82,108.39,107.98,62.90,62.07,56.37,50.78,50.60,48.68$, 48.47, 40.69, 25.69, 21.66, 18.13, 9.80, 9.58. MS-ESI $(\mathrm{m} / \mathrm{z}): 561.44(\mathrm{M}+\mathrm{H})^{+}$.HRMS-ESI $(\mathrm{m} / \mathrm{z})$ : $\mathrm{C}_{26} \mathrm{H}_{34} \mathrm{O}_{7} \mathrm{~N}_{6} \mathrm{~F}$, Calcd: $561.24675(\mathrm{M}+\mathrm{H})^{+}$; Found: $561.24691(\mathrm{M}+\mathrm{H})^{+}$.

\subsection{MIC Determination}

All compounds were screened for their in vitro antibacterial activity against representative Grampositive and Gram-negative strains, by means of standard twofold serial dilution method using agar media [17]. Minimum inhibitory concentration (MIC) is defined as the minimum concentration of the compound required to give complete inhibition of bacterial growth after incubation at $35^{\circ} \mathrm{C}$ for $18-24 \mathrm{~h}$.

\section{Conclusions}

In summary, a series of amino acid and dipeptide prodrugs of IMB-070593 were designed, synthesized and evaluated for their water solubility and antibacterial activity in this study. Our results reveal that the solubility $(>85 \mathrm{mg} / \mathrm{mL}$ ) of four amino acid prodrugs $4 \mathbf{a}, \mathbf{b}, \mathbf{e}, \mathbf{f}$ and two dipeptide prodrugs $\mathbf{4 k}$,l was much greater than that of IMB-070593 mesylate $(22.5 \mathrm{mg} / \mathrm{mL})$. Compounds $4 \mathbf{a}$ and $4 \mathbf{k}$ were found to have lower $\mathrm{ED}_{50}$ values when administered by intravenous injection, compared with orally. Moreover, both of them show stronger efficacy against drug-sensitive Gram-positive strains than the parent drug, as opposed to Gram-negative and drug-resistant Gram-positive strains.

\section{Acknowledgments}

This work was supported by the National S\&T Major Special Project on Major New Drug Innovations (2012ZX09301002-001-017/023, 2014ZX09507009-003) and NSFC 81373267-003.

\section{Author Contributions}

Conceived and designed the experiments: Mingliang Liu, Huiyuan Guo. Performed the experiments: Tingting Zhang, Jinwei Wu and Kaixiang Liu. Analyzed the data: Tingting Zhang, Jinwei $\mathrm{Wu}$, Shihong Chen, Kaixiang Liu and Yabin Lin. Wrote the paper: Tingting Zhang, Mingliang Liu, Huiyuan Guo. All authors read and approved the final manuscript. 


\section{Conflicts of Interest}

The authors declare no conflict of interest.

\section{References and Notes}

1. Feng, L.S.; Liu, M.L.; Wang, S.; Chai, Y.; Lv, K.; Shan, G.Z.; Cao, J.; Li, S.J.; Guo, H.Y. Synthesis of naphthyridone derivatives containing 8-alkoxyimino-1,6-dizaspiro[3.4]octane scaffolds. Tetrahedron 2011, 67, 8264-8270.

2. Liu, M.L.; Guo, H.Y. Evolution of quinolone antibacterial drugs. World. Notes. Antibiot. (Chin.) 2006, 27, 69-75.

3. Dang, Z.; Yang, Y.S.; Ji, R.Y.; Zhang, S.H. Synthesis and antibacterial activity of novel fluoroquinolones containing substituted piperidines. Bioorg. Med. Chem. Lett. 2007, 17, 4523-4526.

4. Hong, C.Y.; Kim, Y.K.; Chang, J.H.; Kim, S.H.; Choi, H.; Nam, D.H.; Kim, Y.Z.; Kwak, J.H. Novel fluoroquinolone antibacterial agents containing oxime-substituted (aminomethyl)pyrrolidines: Synthesis and antibacterial activity of 7-(4-(aminomethyl)-3-(methoxyimino)pyrrolidin-1-yl)-1cyclopropyl-6-fluoro-4-oxo-1,4-dihydro[1,8]naphthyridine-3-carboxylic acid (LB20304). J. Med. Chem. 1997, 40, 3584-3593.

5. Choi, D.R.; Shin, J.H.; Yang, J.; Yoon, S.H.; Jung, Y.H. Syntheses and biological evaluation of new fluoroquinolone antibacterials containing chiral oxiimino pyrrolidine. Bioorg. Med. Chem. Lett. 2004, 14, 1273-1277.

6. Kwon, A.R.; Min, Y.H.; Ryu, J.M.; Choi, D.R.; Shim, M.J.; Choi, E.C. In vitro and in vivo activities of DW-224a, a novel fluoroquinolone antibiotic agent. Chin. J. Antimicrob. Chemother. 2006, 58, 684-688.

7. Lv, K.; Sun, Y.X.; Sun, L.Y.; Wei, Z.Q.; Guo, H.Y.; Wu, J.W.; Liu, M.L. Design, synthesis, and in vitro antibacterial activity of fluoroquinolone derivatives containing a chiral 3-(alkoxyimino)-2(aminomethyl)azetidine moiety. Chem. Med. Chem. 2012, 7, 1230-1236.

8. Lv, K.; Liu, M.L.; Feng, L.S.; Sun, L.Y.; Sun, Y.X.; Wei, Z.Q.; Guo, H.Y. Synthesis and antibacterial activity of naphthyridone derivatives containing mono/difluoro-methyloxime pyrrolidine scaffolds. Eur. J. Med. Chem. 2012, 47, 619-625.

9. Wang, X.Y.; Guo, Q.; Wang, Y.C.; Liu, B.Q.; Liu, M.L.; Sun, L.Y.; Guo, H.Y. Synthesis and antibacterial activity of 7-(3-amino-4-alkoxyimino-1-piperidyl)quinolones. Acta Pharm. Sin. 2008, 43, 819-827.

10. Zhang, Y.B.; Li, G.Q.; Liu, M.L.; You, X.F.; Feng, L.S.; Lv, K.; Cao, J.; Guo, H.Y. Synthesis and in vitro antibacterial activity of 7-(3-alkoxyimino-5-amino/methylaminopiperidin-1-yl)fluoroquinolone derivatives. Bioorg. Med. Chem. Lett. 2011, 21, 928-931.

11. Guo, H.Y.; Liu, M.L.; Liu, B.Q.; Hu, J.S.; Wu, J.W.; Wang, Z. Application of 7-(4-alkoxyimino3-aminopiperidin-1-yl)fluoroquinolones and their combinations. CN Patent 101863876, 20 October 2010.

12. Sanchez, J.P.; Domagala, J.M.; Heifetz, C.L.; Priebe, S.R.; Sesnie, J.A.; Trehan, A.K. Quinolone antibacterial agents. Synthesis and structure-activity relationships of a series of amino acid 
prodrugs of racemic and chiral 7-(3-amino-1-pyrrolidinyl)quinolones. Highly soluble quinolone prodrugs with in vivo pseudomonas activity. J. Med. Chem. 1992, 35, 1764-1773.

13. De Clercq, E. Dancing with chemical formulae of antivirals: A personal account. Biochem. Pharm. 2013, 86, 711-725.

14. Liu, M.L.; Guo, H.Y. Synthesis and in vivo antibacterial activity of tosufloxacin prodrugs. Chin. J. Antibiot. (Chin.) 2004, 29, 590-591.

15. Liu, M.L.; Wei, Y.G.; Sun, L.Y.; Zhang, S.H.; Guo, H.Y. Synthesis and the in vivo and in vitro antibacterial activity of moxifloxacin prodrugs. Chin. J. Antibiot. (Chin.) 2003, 28, 647-653.

16. Furfine, E.S.; Baker, C.T.; Hale, M.R.; Reynolds, D.J.; Salisbury, J.A.; Searle, A.D.; Studenberg, S.D.; Todd, D.; Tung, R.D.; Spaltenstein, A. Preclinical pharmacology and pharmacokinetics of GW433908, a water-soluble prodrug of the human immunodeficiency virus protease inhibitor amprenavir. Antimicrob. Agents. Chemother. 2004, 48, 791-798.

17. MIC values were determined as described by the NCCLS (see: National Committee for Clinical Laboratory Standards, Performance Standards for Antimicrobial Susceptibility Testing: 11th Informational Supplement, Volume 21; NCCLS: Wayne, PA, USA, 2001, M100-S11). MIC was defined as the lowest concentration of each compound that inhibits visible growth of bacteria after incubation at $35^{\circ} \mathrm{C}$ for $18-24 \mathrm{~h}$.

18. Jahani, F.; Tajbakhsh, M.; Golchoubian, H.; Khaksar, S. Guanidine hydrochloride as an organocatalyst for $N$-Boc protection of amino groups. Tetrahedron. Lett. 2011, 52, 1260-1264.

19. Ling, Y.; Ye, X.L.; Ji, H.; Zhang, Y.H.; Lai, Y.S.; Peng, S.X.; Tian, J.D. Synthesis and evaluation of nitric oxide-releasing derivatives of farnesylthiosalicylic acid as anti-tumor agents. Bioorg. Med. Chem. 2010, 18, 3448-3456.

20. Sutherland, A.; Willis, C.L. Chemoenzymatic synthesis of 4-amino-2-hydroxy acids: A comparison of mutant and wild-type oxidoreductases. J. Org. Chem. 1998, 63, 7764-7769.

21. Gowda, D.C.; Abiraj, K.; Augustine, P. New hydrogenation catalyst: An advantageous method for the removal of hydrogenolysable protecting groups in peptide synthesis. Lett. Pept. Sci. 2002, 9, $43-47$.

22. Otsuka, H.; Inouye, K.; Kanayama, M.; Shinozaki, F. The synthesis and biological activities of a hexadecapeptide corresponding to a modified sequence in the corticotropin structure. Bull. Chem. Soc. Jpn. 1965, 38, 679-680.

23. Barcelo, G.; Senet, J.P.; Sennyey, G.; Bensoam, J.; Loffet, A. Alkyl 1-chloroalkyl carbonates: Reagents for the synthesis of carbamates and protection of amino groups. Synthesis (Stuttg.). 1986, 627-623.

Sample Availability: Samples of the compounds $\mathbf{3 b}-\mathbf{j}, \mathbf{3 l}$ and $\mathbf{4 a}-\mathbf{i}$ and $4 \mathbf{k}$ are available from the authors.

(C) 2014 by the authors; licensee MDPI, Basel, Switzerland. This article is an open access article distributed under the terms and conditions of the Creative Commons Attribution license (http://creativecommons.org/licenses/by/3.0/). 